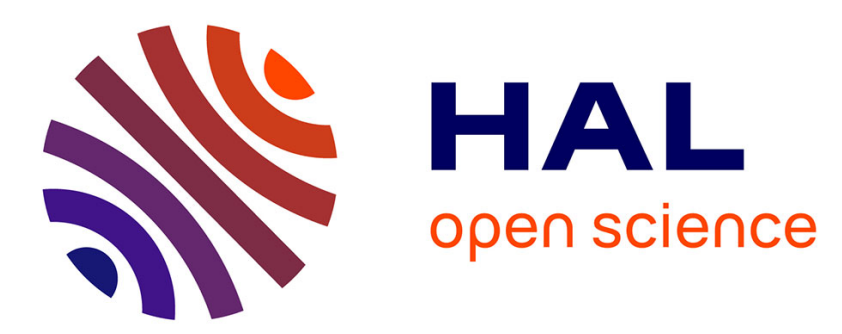

\title{
Piezo-electromechanical smart materials with distributed arrays of piezoelectric transducers: Current and upcoming applications
}

Ivan Giorgio, Luca Galantucci, Alessandro Della Corte, Dionisio del Vescovo

\section{- To cite this version:}

Ivan Giorgio, Luca Galantucci, Alessandro Della Corte, Dionisio del Vescovo. Piezo-electromechanical smart materials with distributed arrays of piezoelectric transducers: Current and upcoming applications. International Journal of Applied Electromagnetics and Mechanics, 2015, 47 (4), pp.1051-1084. 10.3233/JAE-140148 . hal-01121358

\section{HAL Id: hal-01121358 \\ https://hal.science/hal-01121358}

Submitted on 28 Feb 2015

HAL is a multi-disciplinary open access archive for the deposit and dissemination of scientific research documents, whether they are published or not. The documents may come from teaching and research institutions in France or abroad, or from public or private research centers.
L'archive ouverte pluridisciplinaire HAL, est destinée au dépôt et à la diffusion de documents scientifiques de niveau recherche, publiés ou non, émanant des établissements d'enseignement et de recherche français ou étrangers, des laboratoires publics ou privés. 


\title{
Piezo-electromechanical Smart Materials with distributed arrays of Piezoelectric Transducers: current and upcoming applications
}

\author{
Ivan Giorgio, Luca Galantuccił, Alessandro Della Corte ${ }^{\ddagger}$ and Dionisio Del Vescovo ${ }^{\S}$
}

December 28, 2014

\begin{abstract}
This review paper intends to gather and organize a series of works which discuss the possibility of exploiting the mechanical properties of distributed arrays of piezoelectric transducers. The concept can be described as follows: on every structural member one can uniformly distribute an array of piezoelectric transducers whose electric terminals are to be connected to a suitably optimized electric waveguide. If the aim of such a modification is identified to be the suppression of mechanical vibrations then the optimal electric waveguide is identified to be the 'electric analog' of the considered structural member. The obtained electromechanical systems were called PEM (PiezoElectroMechanical) structures. The authors especially focus on the role played by Lagrange methods in the design of these analog circuits and in the study of PEM structures and we suggest some possible research developments in the conception of new devices, in their study and in their technological application. Other potential uses of PEMs, such as Structural Health Monitoring and Energy Harvesting, are described as well. PEM structures can be regarded as a particular kind of smart materials, i.e. materials especially designed and engineered to show a specific and well-defined response to external excitations: for this reason, the authors try to find connection between PEM beams and plates and some micromorphic materials whose properties as carriers of waves have been studied recently. Finally, this paper aims to establish some links among some concepts which are used in different cultural groups, as smart structure, metamaterial and functional structural modifications, showing how appropriate would be to avoid the use of different names for similar concepts.
\end{abstract}

Keywords: Piezoelectric transducers; vibration and sound radiation control; electric analog; structural health monitoring; energy harvesting.

\section{General picture}

The aim of this paper is simply stated: to attract the attention of the (nowadays numerous) researchers in the field toward the use of distributed arraus of piezoelectric transducers, which has, in our opinion, plenty of prospects for future theoretical and practical issues.

${ }^{*}$ Dep. of Mechanical and Aerospace Engineering, SAPIENZA Università di Roma, via Eudossiana 18, 00184 Rome, Italy; Int. Research Center for the Mathematics and Mechanics of Complex Systems (MeMoCS), Palazzo Caetani, 04012 Cisterna di Latina, Italy.

${ }^{\dagger}$ Politecnico di Milano, Department of Structural Engineering DIS, Italy; Int. Research Center for the Mathematics and Mechanics of Complex Systems (MeMoCS), Palazzo Caetani, 04012 Cisterna di Latina, Italy.

${ }^{\ddagger}$ Dep. of Mechanical and Aerospace Engineering, SAPIENZA Università di Roma, via Eudossiana 18, 00184 Rome, Italy

$\S$ Dep. of Mechanical and Aerospace Engineering, SAPIENZA Università di Roma, via Eudossiana 18, 00184 Rome, Italy; Int. Research Center for the Mathematics and Mechanics of Complex Systems (MeMoCS), Palazzo Caetani, 04012 Cisterna di Latina, Italy. 
Indeed it seems that, notwithstanding the remarkable research efforts of a rather large group of persons, the concept of control via a distributed array of piezoelectric transducers interconnected by electric passive waveguides (see e.g. the papers $[67,208,1]$ ) is not yet exploited to its full potential.

The use of piezoelectric materials is of primary relevance for theoretic and applied mechanics and widespread in the literature. The piezoelectric elements are indeed powerful tools in today applied mechanics, as they have the remarkable properties of generating an electric charge when subjected to mechanical strains (the so called direct piezoelectric effect) and vice-versa of generating a local deformation when a charge or a voltage is applied (the inverse piezoelectric effect). Moreover, one single transducer can be used as co-located sensor-actuator pair, i.e. it is capable, at the same time, of measuring a quantity and acting on it (or another one) in the same location. When an electronic circuit is connected to such a transducer, bonded to the structure, the dynamic of the circuit influences that of the mechanical system and vice-versa.

The most recent developments of the technology of piezoelectromechanical transducers supply for the design of smart structures for very powerful actuating devices.

Regarding vibration control using piezoelectric transducers, several strategies of have been proposed, the main of which being:

- Active control circuits: the signal initially read by sensor is subsequently processed, amplified, elaborated by the control circuit and then sent to the actuators.

- Passive and semi-passive control circuits: passive control strategies are obtained by shunting the piezoelectric transducers with an optimized network of optimized passive components.

Each of these approaches has its advantages and drawbacks. The active control systems can be very effective, but as a drawback they require high power electronics and, since they supply power to the mechanical system, they can cause instability. The passive control circuits cannot supply power to the system, and so cannot cause instability. However very often this control networks are too complex for a 'real' discrete passive synthesis, or the designed nominal value of their electric components is very large. As a consequence they need to be realized using active components, while the control law is still remaining passive. This technique, called 'virtual passive (or semi-passive) approach', implements in an active manner the behavior of passive damping systems. It is made possible by the collocated nature of the piezoelectric transducers, see [145, 204]. Besides, it is argued in [144] that the shunt damping technique can be interpreted as a multi-variable feedback control problem, in which the impedance, or alternatively the admittance of the electrical multi-port shunt, constitutes the feedback controller. Within the framework of semi-passive approach for structural vibration damping, the synchronized switch damping (SSD) technique, which is based on a probabilistic description of the piezovoltage, can also be mentioned (see e.g. [114]). A variation of this idea is the so-called self-powered synchronized switch damping on inductor (SSDI), which relies on switching intermittently the piezoelement on a resonant circuit ([120]); a similar idea was already introduced and studied in ([45]), and then extended to multi-modal vibration damping ([46]).

It is now possible (see e.g. [205]) to include in piezoelectromechanical structures some Piezoelectric Transducers, namely PZT (ceramic perovskite materials that show significant piezoelectric effects) whose performances were unparalled only few years ago ([158]): indeed the nowadays available PZTs are able to exert forces up to a nominal value of $1000 \mathrm{~N}$ as a response of the application of $1000 \mathrm{~V}$. Moreover the maximal deformation which can be sustained by the aforementioned transducers without being damaged amounts to $30 \mu \mathrm{m}$ and their resistance to fatigue and crack growth has been dramatically improved.

The actuator properties of piezoceramic transducers are essentially described by two parameters: the blocking force $F_{B}$ and the free displacement, $S_{0}$. When a voltage $U$ is applied to the free (unblocked) actuator, it reaches its maximum displacement $S_{0}$. The force required to prevent any length change at all 
is called the blocking force, $F_{B}$. Some characteristic values these parameters are: $U$ from -250 to $1000, F_{B}$ $\simeq 1000 \mathrm{~N}$, and $S_{0} 800 \mu \mathrm{m} / \mathrm{m}[205]$.

However (see the corresponding subsection in the section relative to the research perspectives which we see attainable in a close future) a lot can be still done to improve the performances of PZTs.

\subsection{The concept of continuously distributed arrays of Piezo-Electric Transduc- ers (PET)}

The energy transduction between mechanical degrees of freedom and electrical degrees of freedom is a concept which is widely used in the literature.

When considering both active and passive control two main strategies are possible: localized and distributed control. The first approach uses a control system with optimally placed piezoelectric patches [116], connected to a circuit operating in selected frequency windows. With respect to the distributed controller this scheme uses a smaller number of piezoelectric elements and, usually, a simpler circuit. Therefore it may introduce a reasonable performance trade-off between the complexity of the controller and the amount of vibrational energy transferred to (and dissipated by) the circuit.

However, less frequently it has been discussed the concept of transduction performed by an array of uniformly distributed PZTs, which, in presence of long wavelength signals, can be modeled as a continuum.

A different approach for solving the same kind of problems considers indeed a distributed piezoelectric network connected to a passive or semi-passive modular circuit, optimized for harvesting mechanical energy of the plate and dissipating it into the non-conservative part of the circuit. The uniform spatial distribution of sensors and actuators allows to have, with optimized electrical parameters, a duality between the electrical circuit and the mechanical structure. This duality has as its consequence the superposition of the electric and mechanic eigenfrequencies, thus allowing an optimal energy flow. This passive technique assures stability and optimizes broadband control.

\subsection{The concept of electric waveguide coupled to mechanical structural elements via PETs}

Once a structural member has been endowed by a continuous array of PETs one can expect to be allowed to interconnect the electric terminals of each of these PETs via a suitable electr(on)ic circuitry.

The following problem naturally arises in this context: which is the optimal electric waveguide to be connected to an array of PETs mechanically connected with a given structural member?

Of course this optimization problem needs to be precisely formulated by specifying the objective function to be minimized.

This can be obviously done in many ways, depending on which kind of performances one wants to require for its PEM structural member. Several answers were given in the literature as it will be discussed in the following sections.

\subsection{Applications}

The potential applications of the ideas described above are, as already stated, very numerous. Among them, Vibration and Sound Radiation Control (VSRC), Energy Harvesting (EH) and Structural Health Monitoring (SHM) are the most likely to be practically relevan in the next future.

The study of vibration control problems is today a very wide and rapidly changing research field in continuum mechanics, and wave propagation is of course a key topic. In particular, passive control of 
vibrations employing distributed piezoelectric transducers is investigated in [72, 63, 209, 73, 176], while active control of vibration and sound transmission is studied in $[188,162]$. However, literature still lacks extensive studies about dissipation of energy connected to wave propagation in PEMS.

Concerning EH, it should be noted that it requires a transduction mechanism to generate electrical energy from motion and a mechanical system that couples environmental displacements to the transduction mechanism [98, 97, 96]. The design of the mechanical system should maximise the coupling between the kinetic energy source and the transduction mechanism, and will depend entirely upon the characteristics of the environmental motion. The mechanical component can be attached to an inertial frame which acts as the fixed reference. The inertial frame transmits the vibrations to a suspended inertial mass producing a relative displacement between them. Such a system would possess a resonant frequency which can be designed to match the characteristic frequency of the application environment. This approach magnifies the environmental vibration amplitude by the quality factor of the resonant system. A vibrating piezoelectric device differs from a typical electrical power source in that its internal impedance is capacitive rather than inductive in nature, and it may be driven by mechanical motion of varying amplitude.

For several years the SHM has been one of the most interesting engineering problems and it has motivated the research on novel technologies and realization of advanced devices. In the literature there are different structural modifications proposed for improving (electro)mechanical systems, in terms of control and efficiency. Direct inspection (e.g. acoustic and ultrasonic measurements, thermal emissions, radiography) is most widely used: this approach requires in general a knowledge of the area where the damage is positioned and the material properties and state fields like displacements, temperature and stress. However, it suffers from problems such as high inspection costs, high dependence on the inspector's experience, considerable time length required, and great difficulty in checking inaccessible locations. The challenge in designing a SHM system is knowing what changes to look for and how to identify them. The characteristics of damage in a particular structure play a key role in defining the architecture of the SHM system. The resulting changes, i.e. damage signature, will dictate the type of sensor that is required, which in turn determines the requirements for the rest of the components in the system. The ability of monitoring the structural health of mechanical systems is becoming increasingly important. A wide variety of effective local non-destructive evaluation tools is available. However, damage identification based upon changes in vibration characteristics is one of the few methods that monitor changes in the structure on a global basis.

Over the last two decades, many automated SHM techniques have been reported in the literature. Global static response techniques, such as the static displacement measurement technique or the static strain measurement technique aim for structural system identification from the static response of structures. However, application of large loads and measuring corresponding deflections or strains (which these techniques require) is not very practical for real-life structures. The global dynamic techniques aim for similar system identification from the dynamic response of structures. However, these techniques rely heavily on modal data pertaining to the first few modes only. These modes, being global, are not sensitive enough to detect localized damage, such as incipient damage in the form of cracks. A common limitation of the global techniques is that they require a high-fidelity model of the structure to start with. They also demand intensive computation to process the measured data. Many investigators have therefore integrated the global techniques with artificial neural networks to non-parametrically predict the damage in an apparent attempt to minimize the a priori information about the structure $[26,178,99,25]$. In $[71,206,75]$ the it was proposed to exploit the electric circuits designed in piezoelectro-mechanic structures (PEMS) for optimizing vibration control also for getting damage monitoring and progress, by means of measurements of the system response to electric impulses.

In [214], a passive stress-sensory network based on distributed piezoelectric circular plates embedded in concrete is employed for concrete health monitoring to determine the structural state in concrete. The structure is mechanically or acoustically excited to cause impact wave or acoustic wave propagation within 
concrete which is sensed by the piezoelectric distributed network.

\section{Piezo-Electric Transducers}

The word transducer indicates a device that can transfer energy between one system and another. In case such a flow of energy is turned towards the system to control and the purpose is to do work on it, the transducer behaves as actuator; on the contrary, if the energy moves the other way round and the purpose is to bring information, then it has the behavior of a sensor.

Piezoelectric materials can be used both as actuators and as sensors because of reversibility of the piezoelectric effect. Thanks to the direct effect a piezoelectric patch is a sensor of strain, in a similar manner of a strain gauge, or of a strain rate; for the converse effect, it is able to impose local deformation by the application of suitable voltage or charge, i.e. it is an actuator. Moreover, the piezoelectric element can be utilized even at the same time in both the ways, making it particularly attractive for many applications.

The linear theory of piezoelectricity is based on two approximations [18]. First, the non-linear theory of electroelasticity is derived from the well-known conservation laws for a mechanical continuum and the conservation laws from Maxwell equations. The quasi-static electric field approximation allows for the electric field $E_{i}$ to be derivable from a scalar potential function $\phi$, i.e. $E_{i}=-\phi_{, i}$. It is also assumed that the magnetic field and magnetization have negligible influence. The second approximation, from which the linear theory of piezoelectricity is derived, assumes that deformations are infinitesimal - the strain tensor is the symmetric portion of the spatial gradient of the mechanical displacement - and that electric fields are small [196, 169]. In that theory, the charge equations of electrostatics are coupled to the mechanical deformations by using a modified Lagrangian density given by

$$
\mathfrak{L}=\frac{1}{2} \rho \dot{u}_{i} \dot{u}_{i}-H\left(S_{i j}, E_{i}\right)
$$

where $H\left(S_{i j}, E_{i}\right)$ is the electric enthalpy density function, which depend on $S_{i j}$, i.e. the components of the strain tensor, and on $E_{i}$, i.e. the components of the electric field vector. The electric enthalpy density [18] can be expressed as

$$
H\left(S_{i j}, E_{i}\right)=\frac{1}{2} \mathfrak{c}_{i j k l}^{E} S_{i j} S_{k l}-e_{r i j} E_{r} S_{i j}-\frac{1}{2} \epsilon^{S}{ }_{i j} E_{i} E_{j}
$$

in which $\mathfrak{c}^{E}, \boldsymbol{e}$ and $\epsilon^{S}$ are respectively the stiffness tensor with constant electric field, the piezoelectric tensor and dielectric permittivity tensor with constant strain. Applying Hamilton's principle, a set of governing equations can be derived for piezoelectric laminae. Using the above definitions, and assuming that there are no body forces, the variation of the action functional based on the Lagrangian density (1) can be computed as

$$
\int_{t_{0}}^{t_{1}} \int_{\mathcal{B}}\left[\rho \dot{u}_{i} \delta \dot{u}_{i}-T_{i j} \delta S_{i j}-D_{i}(\delta \phi)_{, i}\right] \mathrm{d} V \mathrm{~d} t+\int_{t_{0}}^{t_{1}} \int_{\partial \mathcal{B}}\left(\tau_{i} \delta u_{i}+q \delta \phi\right) \mathrm{d} S \mathrm{~d} t=0
$$

where $T_{i j}$ and $D_{i}$ are the components of the stress tensor and the electric displacement vector, respectively, obtained from the enthalpy $H\left(S_{i j}, E_{i}\right)$ as

$$
T_{i j}=\frac{\partial H}{\partial S_{i j}}=\mathfrak{c}^{E}{ }_{i j k l} S_{k l}-e_{r i j} E_{r}
$$

and 


$$
D_{i}=-\frac{\partial H}{\partial E_{i}}=e_{i k l} S_{k l}+\epsilon^{S}{ }_{i j} E_{j}
$$

In Eq. (3) the mechanical and electric work done by the surface traction $\tau_{i}$ and the applied charge density $q$ have been included. The entire surface of the dielectric is assumed covered with an electrode. Hence $q$ represents the charge density that can exist in the conductor at a dielectric conductor interface. An alternate and equivalent form of the constitutive equations is

$$
\begin{aligned}
& T_{i j}=\mathfrak{c}^{D}{ }_{i j k l} S_{k l}-h_{r i j} D_{r} \\
& E_{i}=-h_{i k l} S_{k l}+\beta^{S}{ }_{i k} D_{k}
\end{aligned}
$$

that is useful in some applications. The relations between the coefficients appearing in the two sets of constitutive equations, Eqs. (4) and (5) and Eqs. (6), may be written

$$
\begin{aligned}
& \mathfrak{c}^{D}{ }_{i j k l}=\mathfrak{c}^{E}{ }_{i j k l}+e_{r i j} h_{r k l} \\
& \beta^{S}{ }_{i k} \epsilon^{S}{ }_{i k}=\delta_{i j}
\end{aligned}
$$

An elementary model of a thin piezoelectric patch surface-bonded on a host beam, considers the transducer to be endowed with a two-fold behavior: (i) from an electrical point of view, it plays the role of a capacitor in parallel connection (series connection) with a current source (voltage source) driven by the mechanical time rate deformation; (ii) from a mechanical point of view, it behaves as a spring with two electrically driven pin forces applied at the patch ends.

By denoting the patch length with $L_{p}$, the stored charge with $Q$, the exerted force with $F_{p}$, the strain with $S_{p}$, and the applied voltage with $V_{p}$, from the above constitutive relations (4), (5) or Eqs. (6), the lumped governing equations can be written in terms of voltage and strain as (see e.g. [43]):

$$
\left\{\begin{array}{l}
Q_{p}=k_{e e} V_{p}+k_{m e} L_{p} S_{p} \\
F_{p}=k_{m m} L_{p} S_{p}-k_{m e} V_{p}
\end{array}\right.
$$

or, in terms of charge and strain as:

$$
\left\{\begin{array}{l}
V_{p}=\hat{k}_{e e} Q_{p}+\hat{k}_{m e} L_{p} S_{p} \\
F_{p}=\hat{k}_{m m} L_{p} S_{p}+\hat{k}_{m e} Q_{p}
\end{array}\right.
$$

in which the following relations between the constitutive parameters can be determined:

$$
\hat{k}_{e e}=\frac{1}{k_{e e}}, \quad \hat{k}_{m e}=-\frac{k_{m e}}{k_{e e}}, \quad \hat{k}_{m m}=\left(k_{m m}+\frac{k_{m e}^{2}}{k_{e e}}\right)
$$

The constitutive equations (8) for the piezoelectric transducer can be physically interpreted from an electrical point of view as a capacitor in parallel connection with a mechanically driven current source (Norton representation); on the other hand, the equations (9) consider the patch as a capacitor in series connection with a mechanically driven voltage source (Thevenin representation).

The sandwich-like structure of the element, in which two thin piezoelectric ceramic sheets are bonded to a center support host structure, provides built-in leverage to amplify the motion and electrical output of the piezoelectric layers. Piezoelectric bending elements are electromechanical transducers that possess high motion and voltage sensitivity. The element structure is shown in Fig. 1. As actuators, the elements can generate large displacements and moderate forces at low levels of electrical drive: one ceramic layer expands laterally and the other layer contracts when an electric field is applied to the element so a bending or 


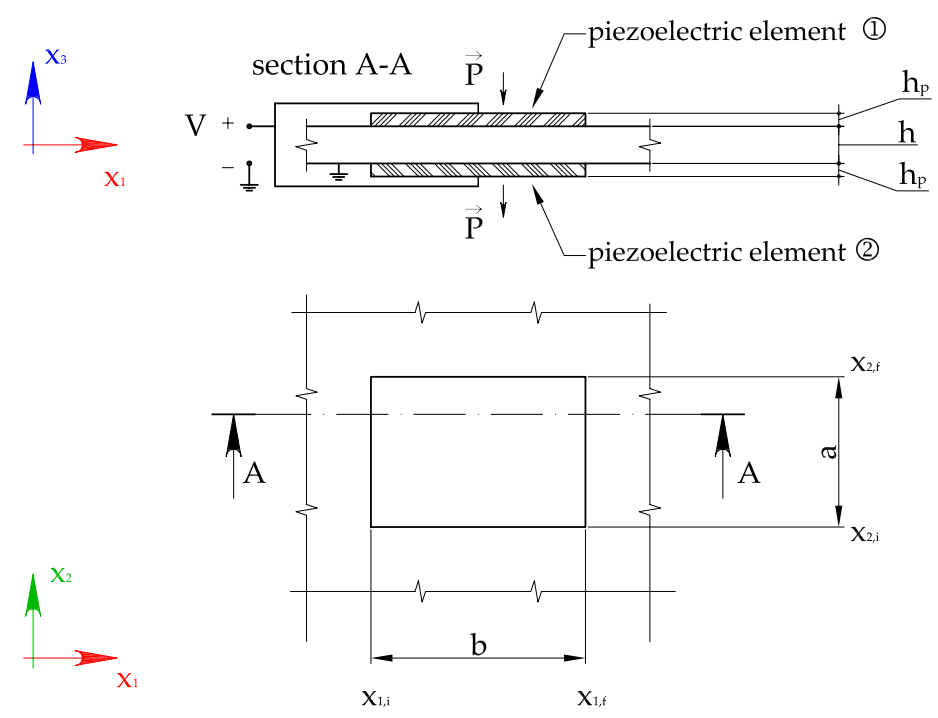

Figure 1: Bending piezoelectric transducer.

deflection of the element places. Typical applications include valves, relays, micro-positioners and vibration controllers. As sensors, the elements can generate electrical signals from sources of low mechanical impedance: the opposing strains result in a bending or deflection of the element that is proportional to the electrode potentials. Typical applications include impact and motion sensors, vibration meters, medical products and industrial sensing devices. The most common bending element shapes are rectangular, square or circular. The piezoelectric layers of a bending element can be electrically connected either in series or in parallel. Piezoelectric polarity orientations and electrical connections for the parallel configuration is shown in the Fig. 1. The series element has one-fourth the capacitance and four times the impedance of the parallel element. As actuators, the parallel element can generate the same deflections and forces as the series element with half the drive voltage. As sensors, the series element has twice the voltage sensitivity of the parallel element. The use of a parallel element is recommended due to drive voltage limitations, on the other hand, the series element can be useful in some applications due to its greater ease of manufacture in not having to access and connect to the center host structure.

\section{Analogical Models and Electrical Analogs of Mechanical Struc- tural Members}

Physical systems which are represented through identical mathematical formalization but have dissimilar physical nature are called analogs (or analogous systems). More in detail, corresponding analogs are characterized by formally coinciding Lagrangian densities and, hence, formally equivalent evolution equations. In general, analogical models, i.e. models exploiting the similarities between analogs, are employed to represent and investigate some physical phenomena, often called the target systems, through other more analyzable and realizable systems. In particular, in the discussion developed in the present work, the concept of "analog" is exploited in order to achieve optimal internal resonance between mechanical structural members and their 


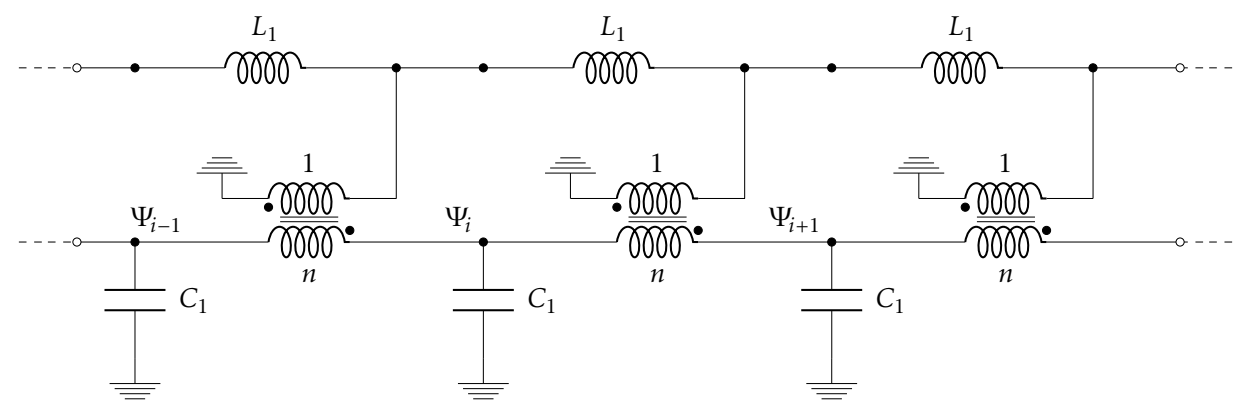

Figure 2: Analog circuit module of an Euler beam corresponding to the voltage-velocity analogy.

corresponding electrical analogs, coupled through the employment of piezoelectric components. The aim of the aforementioned resonance is the control of mechanical members via (see section 4) i) transduction of the mechanical energy possessed by the structural member to an electric form by means of piezoelectric actuators; ii) design of a suitable electric analog; iii) dissipation of electric energy via suitable dissipative circuital elements. In the following paragraphs, the conception of electrical analogs of Euler Beams (3.1) and Kirckhhoff-Love Plates (3.2) will be deeply addressed.

\subsection{Electric Analog of discretized Euler Beams}

In order to synthesize electrical analogs for the Elastica, different procedures can be used, each of them leading, in general, to a particular circuital realization. The key idea is: once a finite differences approximation for the mechanical impedance matrix of a beam element has been found, one well-established synthesis technique (see e.g. [1]) requires paralleling the velocity with the voltage, and the contact actions with the currents.

The motion equation of the analog circuit sketched in Fig. 2 in terms of the flux linkage $\Psi_{i}$ at the generic node $i$, defined as the time integral of the voltage drop across the $i$-th capacitor, can be written as:

$$
\ddot{\Psi}_{i}+\frac{\Psi_{i+2}-4 \Psi_{i+1}+6 \Psi_{i}-4 \Psi_{i-1}+\Psi_{i-2}}{L_{1} C_{1} n^{2}}=0
$$

which represents a discrete form of the Elastica, once the condition

$$
C_{1} L_{1} n^{2}=\frac{\rho}{K_{M}}(\delta l)^{4}
$$

is satisfied [165] introducing the inductance $L_{1}$, the capacitance $C_{1}$ and the turns-ratio of the used transformers $n$ and where $\rho$ the mass per unit length, $K_{M}$ the bending stiffens, $\delta$ indicates the dimensionless spatial sampling step and $l$ is the beam length.

Alternatively, changing the perspective, it is possible consider the identification of the mechanical velocities with electric currents and mechanical contact actions with electric voltages. With the same condition, the governing equation of an electric circuit analogous to discretized Euler Beams, in terms of the time integral of the current flowing through the floating inductors, say $Q_{i}$, as depicted in Fig. 3, is: 


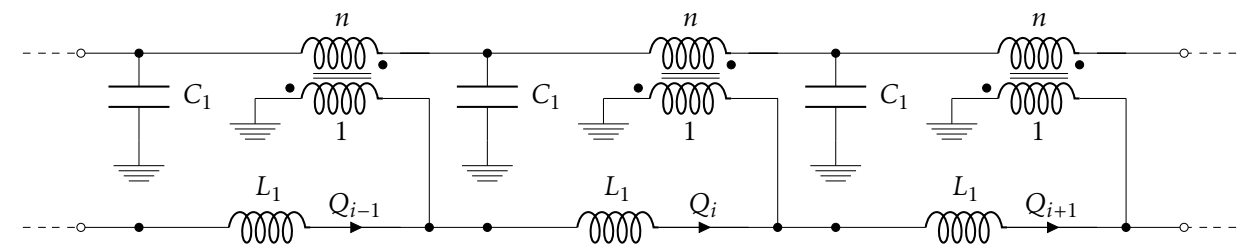

Figure 3: Analog circuit module of an Euler beam corresponding to the current-velocity analogy.

$$
L_{1} \ddot{Q}_{i}+\frac{Q_{i+2}-4 Q_{i+1}+6 Q_{i}-4 Q_{i-1}+Q_{i-2}}{C_{1} n^{2}}=0
$$

The circuit topology in Figures 2 and 3 are completely equivalent; nevertheless, the resulting analog circuits of a given constrained Euler beam are different. This difference lies in the boundary modules which, for the two proposed synthesis solutions, result in totally different circuital constraints. In fact, an electrical node which is short-circuited to ground for one solution results in an open-circuited terminal for the other, and viceversa.

\subsection{Electric Analog of discretized Kirckhhoff-Love Plates}

In order to find an electric circuit analog of a Kirchhoff-Love $(\mathrm{K}-\mathrm{L})^{1}$ plate consisting of completely passive elements, it is possible to consider a discrete Lagrangian density $\mathscr{L}_{m}^{d}$ describing the motion of the K-L plate - in an approximate way - and then to look for an electric circuit that has the same discrete Lagrangian function by identifying the strain mechanical energy, $\mathcal{U}_{m}^{d}$, with the magnetic energy associated with inductors, $\mathcal{U}_{e}$, and the kinetic energy, $\mathcal{K}_{m}^{d}$, with the electric energy associated with capacitors, $\mathcal{K}_{e}$ (see e.g. [4]). Let the square region $\Omega, 0 \leqslant x \leqslant l, 0 \leqslant y \leqslant l$, be covered by a grid with sides parallel to the coordinate axis and grid spacings such that $\Delta x=\Delta y=\delta$. The generic field $f(x, y)$ at any point of the grid will be denoted by the notation:

$$
\begin{aligned}
& f_{i, j}=f\left(x_{i}, y_{j}\right) \\
& x_{i}=i \delta \\
& y_{j}=j \delta
\end{aligned} \quad \text { with } i=1, \ldots N \text { and } j=1, \ldots N
$$

A second-order finite difference discretization of the deformation energy $\mathcal{U}_{m}^{d}$ and the kinetic energy $\mathcal{K}_{m}^{d}$ at any interior node is:

$$
\begin{aligned}
\mathcal{U}_{m}^{d} & =\frac{k_{B}}{2}\left(\frac{u_{0}}{\delta}\right)^{2} \sum_{i} \sum_{j}\left[\left(\Delta_{x x} \tilde{u}\right)_{i, j}^{2}+\left(\Delta_{y y} \tilde{u}\right)_{i, j}^{2}+2 \nu\left(\Delta_{x x} \tilde{u}\right)_{i, j}\left(\Delta_{y y} \tilde{u}\right)_{i, j}+2(1-\nu)\left(\Delta_{x y} \tilde{u}\right)_{i, j}^{2}\right] \\
\mathcal{K}_{m}^{d} & =\frac{\rho h}{2}\left(\frac{u_{0} \delta}{t_{0}}\right)^{2} \sum_{i} \sum_{j}(\dot{\tilde{u}})_{i, j}^{2}
\end{aligned}
$$

\footnotetext{
${ }^{1}$ The K-L plate assumption states that the fibers remain perpendicular to the mid-surface of the plate and maintain their length.
} 


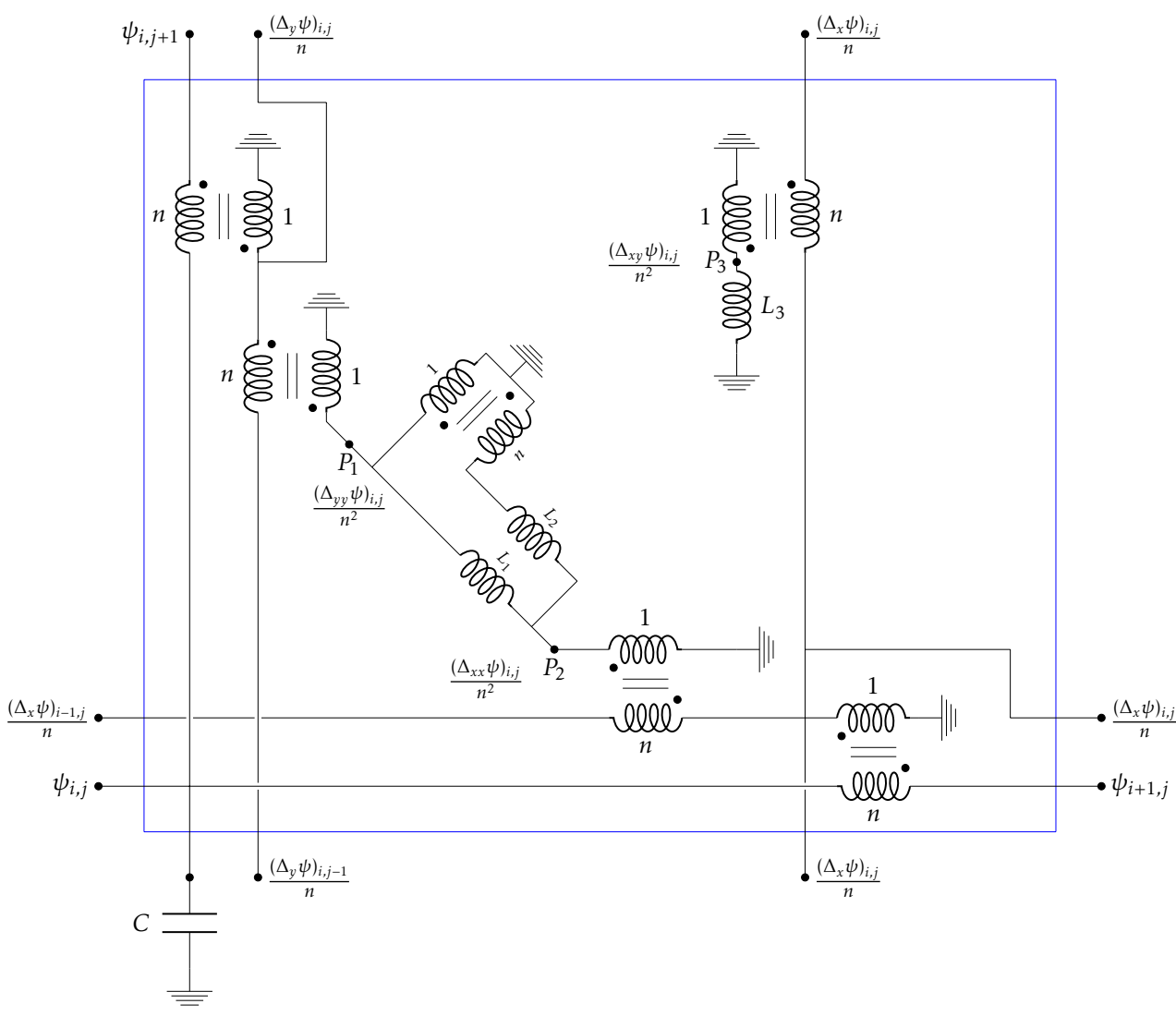

Figure 4: Analog circuital module of the K-L plate.

where the dimensionless variables of deflection $\tilde{u}=u / u_{0}$ and time $\tilde{t}=t / t_{0}$ are introduced by means of the characteristic values of deflection $u_{0}$ and time $t_{0}$. Moreover, $k_{B}=h^{3} Y /\left[12\left(1-\nu^{2}\right)\right]$ denotes bending stiffness, $h$ is the plate thickness, $\rho$ is the mass density and $Y$ and $\nu$ are Young's Modulus and Poisson's ratio, respectively.

Given a quadratic finite dimensional Lagrangian, say $\mathscr{L}_{m}^{d}=\mathcal{K}_{m}^{d}-\mathcal{U}_{m}^{d}$, it is well known (see, e.g., [50]) that it is possible to synthesize a lumped circuit governed by the same evolution (Euler-Lagrange) equations. The resulting analog circuit is in general not topologically equivalent and may have more nodes than the grid. The synthesis can be done, once the K-L plate deflection is recognized as the analog of the flux-linkages of suitable nodes in the electric circuit. Thus, to each node of the sampling grid previously introduced, we will associate a node of the analog circuit, denoted by $n_{i, j}$ and called principal. The flux-linkage $\psi_{i, j}$ will correspond to the deflection $u_{i, j}$. Hence, connecting every node to ground by means of a capacitor, a subcircuit of the analog circuit in which the capacitive electric energy $\mathcal{K}_{e}$ is paralleling the mechanical kinetic energy $\mathcal{K}_{m}^{d}$ is determined. Consequently, the elastic energy $\mathcal{U}_{m}^{d}$ of the plate may be paralleled by the electric energy $\mathcal{U}_{e}$ stored in the remaining part of the analog circuit constituted by a set of inductors opportunely connected to the capacitors by means of an appropriate network of electric transformers. The transformers 
do not store energy, they simply relate the voltages of non-principal nodes to principal ones. Introducing transformers allows to have the second order differences of the displacement fields of Eqs. (14). In Fig. (4) one of the possible topologies for the circuital module (corresponding to the $(i, j)$ node) is presented, that is able to realize an electric analog for the K-L plate as well as the connection among different modules. The electromechanical analogy will be fully accomplished once a dimensionless flux-linkage $\tilde{\psi}=\psi / \psi_{0}$ is introduces, through a characteristic flux-linkage $\psi_{0}$. Consequently, we can express the electric energies $\mathcal{U}_{e}$ and $\mathcal{K}_{e}$ in the following form:

$$
\begin{aligned}
\mathcal{U}_{e} & =\frac{1}{2 n^{4}} \frac{L_{1}+L_{2}}{L_{1} L_{2}} \psi_{0}^{2} \sum_{i} \sum_{j}\left[\left(\Delta_{x x} \tilde{\psi}\right)_{i, j}^{2}+\left(\Delta_{y y} \tilde{\psi}\right)_{i, j}^{2}+2 \frac{L_{1}-L_{2}}{L_{1}+L_{2}}\left(\Delta_{x x} \tilde{\psi}\right)_{i, j}\left(\Delta_{y y} \tilde{\psi}\right)_{i, j}+\frac{L_{1} L_{2}}{L_{3}\left(L_{1}+L_{2}\right)}\left(\Delta_{x y} \tilde{\psi}\right)_{i, j}^{2}\right] \\
\mathcal{K}_{e} & =\frac{C}{2}\left(\frac{\psi_{0}}{t_{0}}\right)^{2} \sum_{i} \sum_{j}(\dot{\tilde{\psi}})_{i, j}^{2}
\end{aligned}
$$

The electric analog circuit is equivalent to the K-L plate once the coefficients of the electric energies (15) are assumed to be equal to the coefficients of the mechanical ones (14). Simple algebra allows us to get the following equalities in which the electric impedances needed in the analog circuit are explicitly given in terms of mechanical parameters and transformers turns-ratios:

$$
\frac{L_{1}}{L_{2}}=\frac{(1+\nu)}{(1-\nu)}, \quad \frac{L_{1}}{L_{3}}=4, \quad n^{4} L_{1} C=\frac{2 \rho h}{k_{B}(1-\nu)} \delta^{4}
$$

From Eqs. (16), it is evident that the choice of the circuital parameters $L_{1}, L_{2}, L_{3}, n$ and $C$ is not unique. The introduction of transformers in the circuital analogs not only allows for the synthesis of completely passive networks (which has not been possible in [1]), but also permits a more ductile variation of the inductance values.

In $[1,2]$ the plate analog circuit is easily derived from the two dimensions centered finite-differences approximation of the Kirchhoff-Love plate equation defined on a uniform-step grid sampling the spatial domain of the plate:

$$
\begin{aligned}
& \left(\frac{16}{\delta^{4}}+\alpha^{4} s^{2}\right) v_{i j}+\frac{v_{i+2 j}-6 v_{i+1 j}-6 v_{i-1 j}+v_{i-2 j}}{\delta^{4}}+\frac{v_{i j+2}-6 v_{i j+1}-6 v_{i j-1}+v_{i j-2}}{\delta^{4}}+ \\
& +\frac{v_{i+1 j+1}+v_{i-1 j+1}+v_{i+1 j-1}+v_{i-1 j-1}}{\delta^{4}}=0
\end{aligned}
$$

in which $s$ is the Laplace-variable and $\alpha$ is a constitutive parameter of the plate, while $v_{i j}$, i.e. the deflection of the K-L plate, can be identified with the flux-linkages of suitable nodes in the electric circuit. Associating each sampling node of the grid to an electrical node of the analog circuit, Eq. (17) can be regarded as the governing equations for the electric potential of the circuit having the structure shown in Fig. 5, where

$$
L_{1}=L / 6 \quad L_{2}=L_{3}=-L
$$

and

$$
C L=\frac{\rho}{k_{B}}(\delta l)^{4}
$$

the presence of negative inductances in the analog circuit forbids its completely passive realization, nevertheless it is easy to electronically simulate a negative inductor using $R C$ active circuits (see e.g. [28]).

Let us underline that the plate circuital analog is not just the superposition of two beam analogs along the axial directions, since two additional transverse transmission lines are necessary $[1,4]$. 


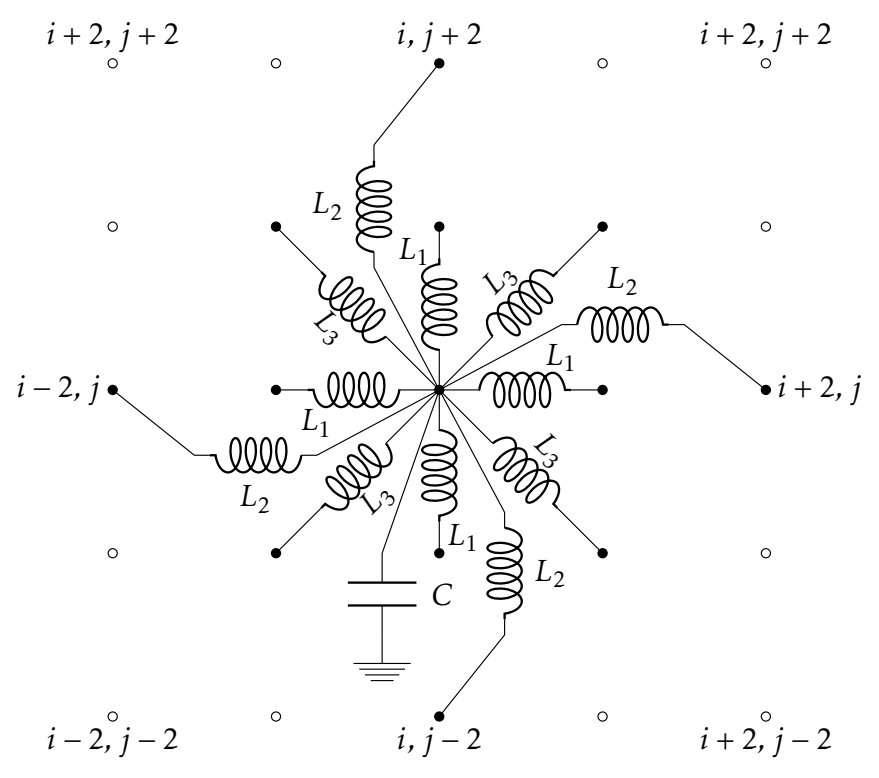

Figure 5: K-L plate analog circuital module.

\section{$4 \quad$ PEM structures as Smart Structures with distributed control}

The concepts of smart structures and smart materials which will be addressed in the present section have been introduced and investigated only recently. Despite ambiguities concerning the specification, the meaning and the use of the adjective smart, it is universally accepted that the latter implies the capacity of a system to self-adapt to a wide class of externally applied actions. In this framework, the intelligence of PEM structures is related to their capability of dumping any kind of external action via the efficient transformation of mechanical energy into electric energy.

The aim in designing PEM structures, which in the present paper are regarded as a particular class of smart structures, consists in the optimization of the dissipation of mechanical vibration energy via the following procedure:

a) a uniformly distributed array of piezoelectric actuators is mechanically connected to a given structural element;

b) these piezoelectric actuators are electrically interconnected via an electric circuit acting as an electric waveguide;

c) this electric waveguide is optimized in order to resonate internally with the original structural member;

d) suitable dissipative circuital elements are introduced in the electric waveguide in order to optimally dissipate the electric energy transduced by the actuators from its original mechanical form. 
Exhaustive theoretical discussions concerning the meaning of the expressions internal resonance and optimal dissipation goes beyond the scope of the present review and, therefore, the authors refer to the following studies $[68,14,137,136,138]$ where the topic is deeply addressed.

The design strategy a) - d) described previously clearly highlights the central role played by the piezoelectric components of PEM structures, i.e. the energy transduction from the mechanical to the electric form, for subsequent energy dissipation by means of suitable dissipative elements introduced in the electronic circuit. The analysis presented in the last cited studies and in [208, 210,67] while exploiting piezoelectric transduction in order to perform a multimodal and passive control of vibrating shells and beams via distributed electronic circuits, show the control efficiency of electric waveguides whose evolution equations coincide with the equations describing the structural member evolution. In fact, the total internal resonance between the mechanical structural member and the coupled electric waveguide is achievable only if the spectral properties of the differential operators governing the evolution of the mechanical and electrical system coincide, i.e. (by the theorem of spectral representation) if the mentioned operators are identical.

The purpose has therefore to be to first design and then tune an electronic circuit possessing eigenfrequencies and eigenmodes coinciding with the ones of the structural member. It is possible to both determine theoretically and realize practically the features of this electronic circuit leading hence to a very effective dissipative electronic waveguide which behaves globally as the electric analog (see section 3) of the corresponding structural member. From a control theory point of view, the introduced electric waveguide can be regarded as a controller which first optimally absorbs the mechanical energy and subsequently dissipates the latter via suitable dissipative elements. On the other hand, from a purely mathematical perspective, it is essential to remark that when the vibration spectra of both the mechanical structure and its electric counterpart coincide, the elctric system can be described as a "mirror system" whose evolving parameter can be regarded as a micro-structural kinematic descriptor. As a consequence, it is possible to state that piezoelectromechanical structures are particular cases of micro-structured continua of the kind studied e.g. by Toupin, Eringen and Mindlin (see [197, 94, 141]).

Despite electric waveguides have been described as purely passive systems, it is worth mentioning that, when active external actions on piezoelectromechanical structures are considered, the stability and bifurcation analysis presents great difficulties, which can be addressed via perturbation methods of the type considered in $[81,173,157,124,38]$. Preliminary studies (reported by Luongo in private communications) suggest in fact that piezoelectromechanical structures are less stable than their purely mechanical counterpart. As a consequence, a stimulating and attractive research direction would be constituted by the conception of a series of theoretical, numerical and experimental investigations aiming at the study and the exploitation of bifurcation analysis of piezoelectromechanical structures, their possible imperfections and their constitutive parameters.

It is worth emphasizing that, in the previuosly outlined investigations, shells or more complex structures were not considered and the analysis was limited to the linear case. As a consequence, a very interesting and stimulating research viewpoint is represented by the investigation of the behavior of piezoelectromechanical structures in the case of nonlinear structural members, which will be addressed in section 6 .

\subsection{Performances of PEM structures}

Research interests and efforts in the design and realization of actual engineering structures exploiting the characteristics of piezoelectronic systems have significantly increased in the last twenty years due to two main factors: (i) the recent technological developments in the production of piezoelectric transducers and (ii) the growing consumers attention towards the control of structural vibrations. This increasing interest in vibration control concerns especially mechanical and aerospace industries which are also interested in secondary benefits 
arising from effective control of structural vibrations, e.g precision in mechanisms maneuvers, reduced fatigue loads, reliability and durability of machinery.

The early studies employing piezoelectric elements in structural control are based on the electronic damping technique, attributed to Olsen [156] and developed subsequently by Swigert and Forward [108]. This technique consists in a closed loop composed of a piezoelectric sensor, converting dynamic strain into electric current, a power amplifier, which amplifies and shifts the electronic current, and a piezoelectric actuator receiving the shifted current. This strategy, soon improved and generalized to the multi-input multi-output case, requiring external power supply, has to be regarded as an active technique, implying that in order to achieve satisfactory results in terms of damping and stability, complex power amplifiers and precise sensing electronics are necessary.

Mainly to overcome the related drawbacks (described before in the Introduction) Hagood and von Flotow [115] proposed a passive shunting technique: a piezoelectric transducer is shunted with a resistor and an inductor, optimally tuned to a structural modal frequency. This control strategy, which electrically parallels the den Hartog theory [117] of tuned-mass damper systems, results to be effective only for the control of a single vibration mode of the structure, i.e. once the mechanical mode is selected the critical inductance, $L_{s}^{*}$, and the optimal position of the piezoelectric patch are univocally determined.

The critical inductance for this lumped control $L_{s}^{*}$ is related to the piezoelectric capacitance $C_{p}$ and to the mechanical modal frequency to be controlled $f_{m}$ by the following relationship $L_{s}^{*} \propto 1 /\left(C_{p} f_{m}^{2}\right)$, imposing severe limitations on the inductances to be used, given the typical values for both $C_{p}$ and $f_{m}$. As a consequence, to achieve the subsequent inductance range $\left(10^{2}-10^{4} \mathrm{H}\right)$ two different techniques can be effectively employed: $(i)$ electronic simulating circuits [28] or (ii) a set of coils wrapped around a magnetic core. However, as stated before, both strategies face serious technological drawbacks: the electronic simulating circuit needs, as in the techniques described previously, an external power supply, while the set of coils adds a significant fraction of the total system mass. In addition, attempting to lower the critical inductance $L_{s}^{*}$ by adding capacitance to the shunting circuit turns out to be ineffective since it necessarily implies a decrease of the efficiency of the piezoelectric coupling.

Some authors extended the technique of the single shunt to damp multiple mechanical modes $[118,218$, 24]. They shunt a single piezoelectric element with a circuit including several resistors, capacitors, and inductors, to obtain a multi-resonant behaviour. Among the different solutions, the current flowing shunt circuit proposed in [24] should be noted for the modular circuital pattern and the reduced number of elements.

In order to overcome all the drawbacks illustrated in the previous paragraph, an enhancement of the design specifications of the control system reveals to be effective. In fact, the idea of an electromechanical analogy arises when imposing the electric controller to be passive and effective on all structural modes. Thus the conceived net-control technique $[207,1,68]$ results in a uniformly distributed array of piezoelectric transducers positioned over the host structure, and an electric passive network, interconnecting the transducers, whose spectral properties are adapted to those of the host structure.

The electric network is designed in order to be resonant at all the structural characteristic frequencies, which allows that its modal shapes can piezoelectrically interact with those of the host structure. These network characteristics ensure the simultaneous control of all vibration modes, without requiring the employment of active elements nor heavy inductors. The synergic behavior of the piezoelectric patches results in high performing energy transduction efficiency over a theoretically infinite frequency bandwidth. Following this approach, but with the aim of reducing the number of PETs, it is worth noting the papers [110, 194]. 


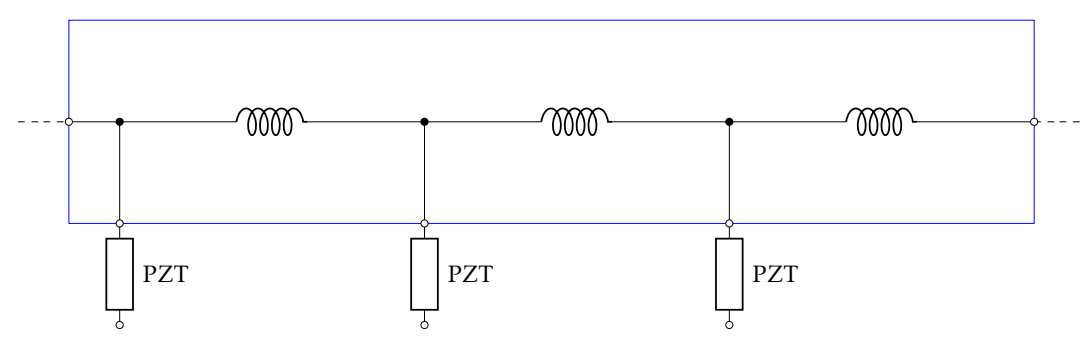

Figure 6: Sketch of a PEM beam with a second-order transmission line.

\subsubsection{Net-controlled structures}

In the framework of net-control strategy, a set of relevant PEM structures is presented in the next paragraphs. In particular, concerning important structural members (e.g. bars, shafts, beams and plates) circuital interconnecting schemes are exhibited and for each structure-circuit pair the basic dynamic properties are addressed. The net-control technique introduced is based on the one-to-one correspondence between the modal frequencies and the modal shapes of the vibrating structure and the interconnecting network, assuring the complete transduction of mechanical into electrical energy.

\subsubsection{Bars and shafts}

The free vibrations of both a bar and a shaft are governed by the so called D'Alembert equation

$$
\ddot{u}=\alpha_{2} u^{\prime \prime}, \quad \text { with } \alpha_{2} \in \mathbb{R}^{+}
$$

where $u$ denotes the kinematical descriptor, namely the axial displacement or the twist angle, and superposed dot and prime mean time and space derivatives, respectively. The electrical distributed circuit governed by the D'Alembert equation is the well-known second-order transmission line. A lumped circuit constituted by a set of $N$ grounded capacitors interconnected via floating inductors does in fact represent a finite difference approximation of the aforementioned distributed circuit.

As a consequence, interconnecting the piezoelectric transducers, bonded over the structure, as the capacitors of the circuit in Fig. 6, the following set of coupled evolution equations governing the free vibrations of the net-controlled bar or shaft is derived

$$
\left\{\begin{array}{l}
\ddot{u}=\alpha_{2} u^{\prime \prime}+\gamma_{2} \dot{\psi}^{\prime} \\
\ddot{\psi}=\beta_{2} \psi^{\prime \prime}-\gamma_{2} \dot{u}^{\prime}
\end{array} \quad \text { with } \alpha_{2}, \beta_{2}, \gamma_{2} \in \mathbb{R}^{+}\right.
$$

$\dot{\psi}$ being the electric voltage measured with respect to a common ground. With reference to Eq. (18), it is sufficient to set $\beta_{2}=\alpha_{2}$ and impose on the electric circuit boundary conditions dual to those of the vibrating structure to achieve the one-to-one correspondence between the mechanical and electrical modal characteristics. On the other hand, the parameter $\beta_{2}$ depends on the value of the line inductance; let $L_{2}^{*}$ be the critical value in order to satisfy the relation $\beta_{2}=\alpha_{2}$. Setting constant the number $N$ of transducers, employing the passive shunting technique (namely, shunting separately each patch with a single inductance), the multi-modal control results to be lost. Let $L_{s 2}^{*}(k)$ be the critical inductance when the passive shunting 
technique is employed to control the $k$-th mode. The ratio $L_{s 2}^{*}(k) / L_{2}^{*}$ results to be proportional to $N^{2} / k^{2}$, [137] implying that for a large number of transducers their interconnection, i.e., the basic concept of PEM systems, sensibly reduces the inductances needed.

\subsubsection{Beams}

The following elastic equation governing the free vibrations of Euler beam

$$
\ddot{u}+\alpha_{4} u^{I V}=0, \quad \text { with } \alpha_{4} \in \mathbb{R}^{+}
$$

does not have a well-known electric analog. Nevertheless, it is possible to couple the beam with the aforementioned second-order transmission line leading to the following set of differential equation [208]:

$$
\left\{\begin{array}{c}
\ddot{u}+\alpha_{4} u^{I V}+\gamma_{3} \dot{\psi}^{\prime \prime}=0 \\
\ddot{\psi}-\beta_{3} \psi^{\prime \prime}-\gamma_{3} \dot{u}^{\prime \prime}=0
\end{array} \quad \text { with } \alpha_{4}, \beta_{3}, \gamma_{3} \in \mathbb{R}^{+}\right.
$$

Similar electrical and mechanical modal shapes could be achieved choosing suitable electric boundary conditions. However mechanical and electrical spectra can not be superimposed since different spectral 'spacings' characterize the latter and, therefore, the line inductance can be eventually tuned to match only one pair of electric and mechanical eigenfrequencies. As in the situation concerning bars and shafts, discussed in paragraph 4.1.2, also when addressing beams, the ratio $L_{s 4}^{*}(k) / L_{3}^{*}(k)$, between the critical inductances required in the passive-shunting and in the second-order net-control technique (used for the $k$-th mode), is proportional to $N^{2} / k^{2}$, clearly highlighting the significant technological advantage when recourse is made to piezoelectric interconnection strategy.

Within the framework illustrated in the previous lines, several approaches have been investigated in order to synthesize the electric analog of the Euler beam. The first strategy proposed and experimentally validated in $[67,1,74]$ envisages the employment of active elements. In particular, in [67] some voltage-driven current sources are used while in [1] the circuital topology is found seeking a finite-differences symmetric scheme of the elastic one and assuming all the used electric elements to be two-terminal networks. This last occurrence necessarily implies the use of negative floating inductances, which must be simulated by electronic active circuits.

Important development towards a feasible passive realization of the beam-analog circuit has been made in [1] and [70], where a circuit constituted by multi-port transformers is obtained relaxing the two-terminal network hypothesis and considering a suitable truncation of the mechanical impedance matrix of a beam element. The main advantage of this last scheme consists in the employment of low inductances, while the substantial drawback lies in the complexity of the circuital topology. A synthesis solution, technologically more convenient in terms of both required inductances and topological complexity, is given in [13] and it is here outlined in Fig. 7. The proposed analog circuit has been synthesized through a finite difference approximation of the infinite dimensional Lagrangian of the Euler beam. The governing equations of the beam net-controlled via the circuits given in $[1,69,13,23]$ are the following:

$$
\left\{\begin{array}{l}
\ddot{u}+\alpha_{4} u^{I V}+\gamma_{4} \dot{\psi}^{\prime \prime}=0 \\
\ddot{\psi}+\beta_{4} \psi^{I V}-\gamma_{4} \dot{u}^{\prime \prime}=0
\end{array} \quad \text { with } \alpha_{4}, \beta_{4}, \gamma_{4} \in \mathbb{R}^{+}\right.
$$

Parameters $\beta_{4}$ and $\gamma_{4}$ in Eq. (20) depend on the adopted electrical scheme and once the parameter $\beta_{4}$ is set equal to $\alpha_{4}$ and the electric boundary conditions have been chosen to be dual of the mechanical ones, the seeked one-to-one correspondence between the electrical and mechanical modal frequencies and modal shapes is established. Taking into account the electric circuit reported in Fig. 7 and setting a unitary turns ratio for 


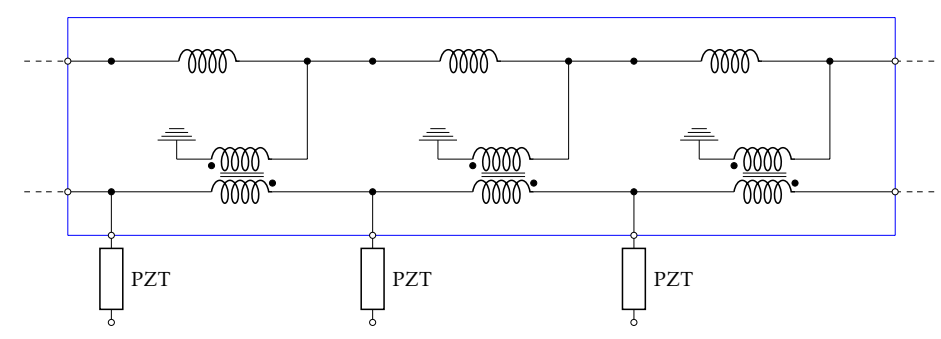

Figure 7: Circuital scheme for a fourth-order interconnection.

the transformers included in the circuit, let $L_{s 4}^{*}(k)$ and $L_{4}^{*}$ be the critical inductances to separately shunt all the $N$ transducers for controlling the $k$-th mode and to equate $\beta_{4}$ to $\alpha_{4}$, respectively: the ratio $L_{s 4}^{*}(k) / L_{4}^{*}$ results to be proportional to $N^{4} / k^{4}$. Therefore, an increase in the number of the piezoelectric patches used in the net-controlled strategy results in a use of extremely advantageous inductances with respect to the passive shunting technique. In addition, the net-controlled beam described by Eqs. (20) also ensures the multi-modal control of the mechanical vibrations through a unique choice of the net inductance $L_{4}^{*}$.

\subsubsection{Plates}

The free vibrations of the Kirchhoff-Love plate are governed by the following differential equation:

$$
\ddot{u}+A_{4} \nabla^{2} \nabla^{2} u=0, \quad \text { with } A_{4} \in \mathbb{R}^{+}
$$

As it occurs for the Euler beam addressed in the previous section, Eq. (21) involves fourth order spatial derivatives and has not well-known electric analogs. As a consequence, the arguments employed in the discussion concerning the Euler beam are exploited for the the conception and realization of the electric analog of the plate structure. As a result, the easiest net-controlled system is realized coupling the Kirchhoff plate with a second-order transmission network, corresponding to the two-dimensional extension of the D'Alembert transmission line. The resulting equations [208] are the following:

$$
\left\{\begin{array}{c}
\ddot{u}+A_{4} \nabla^{2} \nabla^{2} u+\Gamma_{3} \nabla^{2} \dot{\psi}=0 \\
\ddot{\psi}-B_{3} \nabla^{2} \psi-\Gamma_{3} \nabla^{2} \dot{u}=0
\end{array} \quad \text { with } A_{4}, B_{3}, \Gamma_{3} \in \mathbb{R}^{+}\right.
$$

For comparison purposes, let $L_{s p}^{*}(h, k)$ be the critical inductance for the separate resonant shunting relative to the $(h, k)$-th plate mode and $L_{3 p}^{*}(h, k)$ be the critical inductance needed in the net-controlled system (22) to control the same $(h, k)$-th mode. Similar arguments with respect to the discussion performed concerning Eq. (19), describing the beam analog circuit, hold for the current system, i.e. a unimodal control is guaranteed employing more advantageous inductances in the net-controlled system, $L_{s p}^{*}(h, k) / L_{3 p}^{*}(h, k) \propto N /\left(h^{2}+k^{2}\right)$.

On the other hand, concerning the problem of realizing the electric analog of the Kirchhoff-Love plate, the latter has been synthesized in [2] and [1] employing active elements to simulate negative floating inductances. Aiming to avoid the use of active elements, for the motivations illustrated in the introduction of 4.1, the one-dimensional circuital topology reported in Fig. 7 may be extended to the two-dimensional case [3, 5]. the evolution of both net-controlled systems is described by the following coupled differential equations: 


$$
\left\{\begin{array}{l}
\ddot{u}+A_{4} \nabla^{2} \nabla^{2} u+\Gamma_{4} \nabla^{2} \dot{\psi}=0 \\
\ddot{\psi}+B_{4} \nabla^{2} \nabla^{2} \psi-\Gamma_{4} \nabla^{2} \dot{u}=0
\end{array} \quad \text { with } A_{4}, B_{4}, \Gamma_{4} \in \mathbb{R}^{+}\right.
$$

This last set of equations (23) ensures the multi-modal control of the plate vibrations through a unique choice of the net inductance $L_{4 p}^{*}$. Setting the turns ratio of the transformers to be unitary for comparison purposes, the ratio $L_{s p}^{*}(h, k) / L_{4 p}^{*}$ becomes proportional to $N^{2} /\left(h^{2}+k^{2}\right)^{2}$ showing, again, that a large number of patches decreases the required inductances.

To summarize, the main difference between the net-control strategy and the passive shunting technique consists in the presence of interconnections between the distributed patches. Within this strategy, conceiving an interconnecting circuit analog to the host mechanical structure, the simultaneous control of all the mechanical modes is performed employing a single choice of the electric parameters. Concerning relevant structural elements, e.g. bars, beams and plates, completely passive analog circuits have been conceived and their performances as electric controller have been compared with those of (i) separated shunted circuits and (ii) second order transmission lines. The crucial points of this comparative study are mainly two: (a) the multi-modal control is possible only using analog circuits and (b) the necessary critical inductances result to be lower in net-controlled systems. In addition, the damping ratios for electromechanical vibrations can be optimized by suitably introducing in the electric controller some resistors (see [137, 14] and Figs. 6 and 7). For instance, in system (20) conceived for beam control, introducing a suitable resistances in parallel connection with the floating ports of the transformers in Fig. 7, the modal damping ratios $\zeta_{k}$ turn out to be independent on the mode number $k$. The expressions for $\zeta_{k}$ are the following:

$$
\zeta_{k}=\frac{\gamma_{4}}{2 \sqrt{\alpha_{4}}}, \quad k=1,2, \ldots
$$

Having illustrated the state of the art of this very promising research branch, the authors of the present work would like to briefly outline the future developments of it, namely: $i$ ) the synthesis of electric analogs for the net-controlling of more complex structures, as frames and trusses with arbitrary shape and shells ii) an accurate analysis of the homogenization procedure lying beneath the proposed field equations for PEM structures iii) the set up of an experimental procedure to validate this approach.

\section{$5 \quad$ PEM structures as Metamaterials}

It is unlikely that natural materials are capable of organizing themselves into very special and microscopically inhomogenous patterns. For instance, even though the scientific community has been aware, since the first serious studies concerning material properties (pioneered by Lamé), that negative Poisson coefficients for isotropic materials were compatible with the energy conservation principle and did not violate any fundamental principle of physics, only recently it has been possible to design and construct isotropic materials whose macroscopic Poisson ratio is indeed negative.

Unlike what happens e.g. in the case of classical Termodynamics, in this case the principles of physics do not intrinsically take into account the probability measure of a certain 'state', but in fact just establish which materials can be potentially engineered, while statistical considerations are necessary for establishing how probable is the natural onset of a material enjoying some peculiar given and desired properties. Usually, once a material is constructed in order to display some specific features and behavior, it is called a metamaterial.

In this framework, PEM structures, constituted by PETs, are a particular example of metamaterials: the PETs transform, in fact, mechanical energy into electric energy, which is then conveyed into a waveguide whose properties are designed in order to assume a desired behavior. For instance, the system designed could 
have as main goal the dissipation of the traveling energy at the maximum possible rate. More in particular, PEM structures can be regarded as metamaterials whose configuration is described by a displacement field and by (for instance) a set of electric potentials. The coupling between the two fields is assured by the PET transduction which can be generally regarded to be conservative.

It is worth emphasizing that conceptually, focusing the attention only on passive electric circuits strongly limits control performances (see e.g. $[164,165,166]$ ). However, while investigating the performances of active systems it is fundamental to recall that active systems of vibration control need energy consumption for dissipating energy (which is not very often desirable) and show very often strong instabilities. In addition, numerical problems could arise from strongly coupling systems involved in the study of metamaterials, as those presented in $[39,40,41,42,112,52]$.

\subsection{Acoustic metamaterials and wave propagation in micromorphic continua}

The concept of metamaterial was first introduced in optics and in electromagnetism to indicate materials showing peculiar optical or electromagnetic permittivity properties. More recently, the interest of physicist and mechanicians have focused on the concept of acoustic metamaterial which has been investigated in several studies: we refer for instance to [83] or [216]. In general, acoustic metamaterials, and the entire class of mechanical metamaterials, are designed to show peculiar (or exotic) response to externally applied loads. As the whole class of metamaterials, mechanical metamaterials are designed by suitably assembling multiple elementary parts which are usually arranged in (quasi-)periodic substructures to show very peculiar and especially designed properties. In fact, the particular shape, geometry, size, orientation and arrangement of their constituting elements can affect for instance the wave propagation of light or sound in a not-alreadyobserved fashion. As a result, material properties which cannot be found in conventional (natural) materials can be effectively created. Particularly promising in the design of metamaterials are micro-structures which present high-contrast in microscopic properties: the treatment of these micro-structures, at a macroscopic level, have been shown to produce generalized continuum models (see e.g. [6, 106, 107, 143, 142]). The aforementioned micro-structures of metamaterials are conceived so that some of the physical micro-properties characterizing their behavior diverge when the size of the REV (Representative Elementary Volume) tends to zero, while simultaneously some other properties are vanishing in the same limit. It is crucial underlying that the standard homogenization techniques need to be modified and/or generalized in order to be adapted to the process of identifying material properties of metamaterials (see e.g. [192, 193, 146, 142, 140, 56, 57]).

As already anticipated, in the scientific community a raising interest concerns a particular sub-class of mechanical metamaterials: the so-called acoustic metamaterials. For instance in the studies by Neff ([149] and Ghiba et al. [111]) a very specific class of acoustic metamaterials is investigated in which some frequency bands result to be forbidden to wave propagation (see also $[163,174]$ ). It is important to emphasize that the mathematical problems studied in $([150,151])$ are the basis of further scientific investigations in this topic. It is in fact clear that the manifold variety of propagating waves which may exist in metamaterials may produce unexpected applications in their design, showing up-to-now unimagined features.

At this stage an important consideration has to be formulated: PEM structures studied in literature up to now possess, as a mechanical counterpart, either a one- or a two-dimensional continuum. The electromechanical waveguide to be coupled to these continua via PETs, have therefore intrinsically the same dimension. It has to be underlined that it could be very useful to consider PEM structures whose mechanical parts are three-dimensional continua and therefore whose coupled waveguide is an intrinsically three-dimensional waveguide. Obviously, in this case also PETs would need to be spatially distributed. In this context, it could be therefore meaningful to consider PEM structures whose mechanical part is already a micromorphic continuum and, in particular, a second gradient continuum. In fact, as already remarked, second gradient 
materials can be seen as a particular limit case of micromorphic media, as they can be obtained from the latter by constraining the micromorphic tensor to be equal to the classical strain tensor (see [27]).

Available results on wave propagation in second gradient elastic media have shown the onset of exotic phenomena primarily related to screening or transmitting properties of interfaces embedded in such media. In particular, it has been shown (see e.g. [61], [163], [175], [174]) that for wave frequencies sufficiently high in order to interact with the underlying microstructure, very interesting screening or transmitting phenomena can be conceived and described. As a consequence, metamaterials designed by coupling second gradient materials with mirror electric waveguides could lead to the subsequent design of technologically relevant devices, e.g. in the field of stealth technology or vibration and acoustic passive control.

Preliminary results have also been obtained on the study of wave propagation in micromorphic media, showing purely mechanical coupling phenomena with internal degrees of freedom and indicating the inhibition of wave propagation in definite circumstances. These frequency-dependent exotic properties may be enhanced by using the concept on which PEM structures are based, i.e. suitably-conceived metamaterials could be used as exotic waveguides allowing to filter and/or switch on and off specific waves.

\subsection{Functional Structural modifications, metamaterials and smart structures.}

The concept of functional structural modification has been introduced originally in solid and structural mechanics but, actually, it appears to possess a wider meaning, especially if related to the concepts of metamaterials and smart structures. At this stage, an attempt to illustrate the existing relationships among these concepts is therefore fundamental in order to establish potential synergies between different research groups which nowadays do not seem to communicate in the most fruitful manner.

A functional structural modification of a given structure is a modification which, without changing the principal uses of the original structure, is designed in order to fulfill a given engineering collateral purpose (see e.g. [31, 33, 32, 35, 30]). The original structure is often called principal: a well-known example of principal structures coincides with the structures employed in space applications for carrying loads or in Robotics for assembling robot parts and which experience forced vibrations. The latter, in absence of damping, could result into damage onset and hence possibly have even a destructive effect.

In the considered example, the structural modification of the principal structure aims at an efficient damping of the undesired vibrations. The modifications involve the coupling of the principal structure with additional devices, which can be either purely mechanical or be based on different physical phenomena. In the context of the present paper, these structural modifications may be obtained ( see e.g [68]) by connecting the considered structural member to arrays of piezoelectric transducers coupled with resonant dissipative electronic circuits.

The expression functional structural modification is usually employed, as said, to describe a purely mechanical additional structure: as an example, it is worth emphasizing that the concept motivating the following papers [33, 31, 51, 36, 37] stems from the thermodynamics and statistical mechanics of irreversible systems, although the theory of mechanical vibration is often considered far from thermodynamics. The aforementioned papers exploit the concepts of irreversibility, Poincaré recurrence time and thermodynamical equilibrium to conceive a technologically feasible damping device to be combined with the principal structure: this designed structure is coupled with another having many internal extra degrees of freedom evolving according to specifically chosen equations. This strategy allows to trap mechanical vibration energy for a very long time in the extra degrees of freedom (as long as lasts the Poincaré recurrence time), even without introducing any dissipative device. In this way it is possible to construct a conservative system behaving as an effective damping system.

At this stage, it clearly emerges that a metamaterial can originate from a functional structural modifi- 
cation: actually, these modifications coincide with the design of a particular type of metamaterial or smart structure (depending on the length scale at which the modification takes place). If the structural modification involves the introduction of devices whose dimensions are small when compared to the characteristic lengths involved in the considered phenomena (e.g. when modifications involve micro-elements which are small if compared to the specific wave-lengths), the resulting object can effectively be considered a metamaterial. On the other hand, when the added device is external or possesses dimensions comparable with the principal structure, the modified structures can be considered smart structures. Therefore, PEM structures, depending on the size of the applied PET transducers, can be considered either new metamaterials, exploiting piezoelectric effects and the basic phenomena governing the behavior of electric circuits, or smart structures, if the added structural modification has macroscopic dimensions.

The design of micro-structured metamaterials, whose material particles are endowed with such 'energy sinks', is therefore an interesting and stimulating challenge and, in addition, these added microstructures can also be somehow regarded as a particular case of structural modifications. The physical nature characterizing these energy sinks may assume different characters, e.g. these sinks may also be related to 'micro-structural' damage evolution (as those described in $[64,142,172,212,215,211,19]$ ). In fact, bodies in which micro-cracks or other damage mechanisms occur can be modeled both by means of discrete methods (in which models are finite dimensional) or by means of homogenized models, in which, however, extra kinematical descriptors need to be introduced. Interesting results in this context can be found e.g. in [170, 171, 215, 142], whose intended final aim is to produce a macroscopic field theory based on the knowledge of the micro-structure of considered mechanical systems. These macroscopic models are coherent with the models described in [94, 93, 95, 90, 91, 181, 182, 119] in which irreversible phenomena involving dissipation of energy must be taken into account by suitably adding dissipative effects, for instance by means of Hamilton-Rayleigh dissipation potentials.

The phenomena of 'material transformation' induced by any form of evolving damage are some-how similar to phase transitions (see e.g. $[55,84,64,65]$ ) but are not reversible (see e.g. [54, 47]). Damage phenomena may be induced by plastic deformation (see e.g. [48, 49, 107]) or by crack growth (see e.g. [15]) and play a very important role in the context of structural modifications aimed to vibration control. The latter can be in fact obtained by directing some of the mechanical energy involved in some 'micro-structural' vibrations which, on their turn, are to be coupled with dissipative phenomena associated for instance to damage or plastic deformation.

According to the aforementioned description, we are therefore suggesting to interpret the results already available in literature as follows: the mechanisms to trap energy in microscopic energy sinks may be based on very different physical principles and phenomena. They can be related to apparently irreversible trapping of kinetic energy in many microscopic degrees of freedom, or they can be related to the coupling of mechanical with electromagnetic phenomena or they can be related to micro-damage or microscopically concentrated phase transitions. In all the illustrated possibilities, the obtained metamaterial will need to be described by a suitable model deduced from generalized continua theories. Until the 'structural' modifications designed within this strategy do not compromise the mechanical properties of the considered systems, they can be indeed fruitfully exploited. In fact, until the micro-damage does not affect the macroscopic overall behavior of the material, this modified structure or material will be still able to effectively sustain the expected applied loads, as the initial design required.

\subsection{A possible application of PEM structures to drive bone growth}

Piezo-electro-mechanical structures may be embedded in prostheses designed for reconstructing and sustaining damaged bone structures. Bones can be considered, by themselves, as smart materials. A bone, indeed, 
is a dynamic system with physiological remodelling, i.e. with removal and growth capabilities which enable it to easily repair small defects. The current generation of implants consists of passive devices that are unable to recognize damages and to exploit the 'smart' capabilities of the bone tissue. A challenging concept is therefore the creation of a 'smart' technology based on electromagnetic stimulation using wireless power supply systems. Specifically, endowing the implant with a grid of piezoelectric elements (both sensors and actuators), it can be possible to monitor the bone damage state and respond appropriately in order to stimulate the desired formation of new bone tissue.

In fact, it is well-known that periodic vibrations induced by time varying externally applied loads may favor the action of actor cells which remodel and optimize bone tissues (see [198], [44]).

The concept which may be employed is based on the possibility of exciting electric vibrations in electric circuits without direct access to the latter, by simply inducing, for instance, time variations of magnetic fields.

The idea, therefore, consists in modifying already available prostheses by adding PETs and by connecting the added array of transducers to suitable excitable electric circuits. The prosthesis will start vibrating when undergoing an externally applied variation of magnetic field in order to actively contribute to the natural remodeling process. Since low magnitude local vibrations was found to be sufficient to drive the remodeling process, the power needed by a smart endoprosthesis should be rather modest [44], which enables the possibility of powering vibrations from mechanical deformations caused by musculoskeletal loading.

To briefly describe this research possibility, we illustrate evidences concerning the mechanical behavior of living tissues and in particular of bones. Scientists investigating living materials quickly discovered that the required conceptual tools were different from those employed in studying non-living materials (see the pioneering work by Wolff [217]). After the studies performed by Wolff, there have been many attempts to model the mechanical behavior of growing tissues: in the context of the investigations addressed in the present work, the attention is here focused on two different research directions. The first of the latter considers the problem of growth of reconstructed bone with the addition of bioresorbable materials (see [122, 129, 130, 17] and references therein). The second branch attempts the description of biological tissues from a mechanical point of view, with a special stress on their 'micro-structural' behavior (see [101, 103, 100, 102, 104] and references therein).

In the context we are referring to in the present work, the aforementioned studies have to be complemented by studies of contact phenomena between prosteses and reconstructed bone and by deeper investigations concerning the effects of mechanical stimulus on the remodeling phenomena in the interior of reconstructed bone.

Very important are, therefore, the studies regarding the mechanically driven stimulus inside a living tissue and the different models which have been proposed in literature for its description. This stimulus may be regarded as a kinematical parameter whose evolution governs bone growth. In this framework, different possible ways are conceivable to describe how the stimulus is produced in a living or reconstructed tissue: in literature no well-established explanation of all mechanisms governing its production and its transmission is available. In fact, while often the stimulus is assumed to be determined by the local state of deformation (and maybe by the time history of this state), there is biological evidence that the stimulus is also affected by the deformation state of neighboring regions of a given material particle of a deformable tissue. This circumstance may play a crucial role in the phenomena of growth occurring in reconstructed bones and necessarily requires to be taken into account. This non-locality is indeed addressed by means of the introduction of suitable integral operators for representing stimulus field.

On the hand, the second research branch previously anticipated concerns models for 'complex' biological tissues: i.e. tissues which have to be considered as composite materials. For instance, there exist tissues having an internal structure in which a porous matrix filled with an interstitial fluid may be observed (see 
the results presented in $[180,132,133,60,167]$ for the flow modeling of an eventually compressible fluid in deformable porous media). This structure is also reinforced by impermeable collagen fibers: the phenomena related to interstitial fluid flow in articular cartilage motivates the modeling of such a system. Many difficult problems arise in this completely new application field of micro-structured continuum mechanics: for instance, the quantification of the undeformed configuration permeability of fibre-reinforced composite materials and the modeling of its eventual modification in the deformed configuration.

Crucial in this context is the analysis of the length scales involved: their possible separation may in fact simplify the modeling procedure. However, different complex homogenization methods have to be used in any circumstance and, very often, the capillary properties of the interstitial fluid may be of relevance: in this case the results developed in $[109,77,78,183,184,185,186,187]$ can be very useful.

Given the above outlined picture, the possible use of PEM structures in this context should look now evident.

\section{Research Perspectives}

The research hints sketched in the present section are limited to some developments of research activities which are already in progress in MeMoCS International Research Center. The experimental part of the proposed investigations, described and sometimes already in progress, are to be considered with great attention, as the sophisticated models introduced are able to forecast phenomena which could not be imagined as possible and therefore excluded in the design of the most standard experimental apparatuses.

\subsection{Design of more complex/general PEM structures}

Films of Polymeric Piezoelectric Polyvinylidene Fluoride (PVDF) proved to be good materials for distributed sensors and actuators in the vibration control of composite shells of arbitrary shapes. In fact, the integration of sensors and actuators into structures is a crucial step to obtain smart systems [168]. Since the first works on a multi-layered shell coupled with an active distributed vibration controller (see e.g. [200, 199, 201, 82, 20, 21]), big efforts were made to realize new piezo-composite structure both for modeling linear [121] and nonlinear [202] behavior. One method to improve the performance is to segment the symmetrically distributed sensor/actuator layers into a number of co-located subsegments [203], displaying the same kind of perspective which was described in the present paper for uniformly distributed array of piezoelectric patches.

According to this point of view, the study of Piezoelectric Fiber Composites (PFC) is by now a challenge that should be tackled for the opportunities that may open up. These composites are made of fibers surrounded by a resin matrix which provides damage protection and support. They are characterized by a high degree of flexibility and can be profitably used for shells of complex geometry (see e.g. [195, 80]). The study of their stability, e.g. those exposed in $[113,124,126,127,128]$ or, for similar problems related to coupled aeroelastic phenomena, vibration analysis and non-linearities, in [159, 160, 155], can be fruitfully used for future research developments.

Finally, the concept of higher gradient or non-local PEM structural members may be developed exploiting the tools of the Peridynamics based on a non-local force interactions (see e.g. [92, 79]).

\subsection{Design of distributed electric sinks}

In some recent papers the concept of apparent damping in linear mechanical systems is exploited to conceive effective vibration dampers: see the conceptual framework established in [29, 33]. 
The basic idea is simple but challenging: it is well-known that in discrete systems with numerous degrees of freedom, even if the Lagrangian is quadratic (i.e. the evolution equations are linear) it is possible to design mechanical energy traps, i.e. degrees of freedom where the mechanical energy may remain for long time intervals. Remark that this statement is not in contradiction with the Poincaré return Theorem, as the Poincaré return time can be designed to be very large in comparison with the characteristic times of considered vibrational phenomena.

The concept which in $[31,32,34,177]$ was proved to be feasible is indeed based on some spectral properties of mechanical oscillators which may be reproduced by means of electronic circuits.

\subsection{Sound and vibration control via the conception of frequency filters or en- hanced damping effects}

The conception of acoustic metamaterials can exploit different phenomena. For a comprehensive description of the models which we consider needed in this context we refer to [135].

If nonlinearities are to be included into the picture then the results described in $[134,148,147]$ can be an useful starting point. Interesting multiple time scale analyses are conceivable to design wave modulations presenting energy transfer from one vibration mode to others and possibly its more effective dissipation (see the methods presented in $[123,125]$ and references therein). The concept to be exploited in this case is the one called 'energy spillover'. In this case the activable degrees of freedom are those described by the micro-structural kinematic descriptors.

Exploiting non-linearity it is possible to design metamaterials in which — for frequencies belonging to some band gaps - there are not propagating waves, due to the coupling phenomena involving micro-structure.

On the other hand it is rather surprising the result announced $[111,149]$ where it is found that for a 'singular' kind of linear micro-structured continuum (considered not admissible by Mindlin) actually it is possible to choose the constitutive parameters in such a way that actually some even wide band gaps arise. The novelty of the result is two-fold: i) the results seem to indicate that non-linearities are not needed for getting such gaps ii) the mathematical results of well-posedness presented by the authors show that the considered materials are most likely feasible.

It has to be investigated the whole set of micro-structured continua and their physical feasibility (also by means of suitable electronic wave-guides). The aforementioned preliminary results just announced probably show an unexpected richness of behavior for Mindlin-type materials which could hide interesting surprises.

Another concept which needs to be investigated concerns the conception of materials undergoing phase transitions when suitably mechanically solicited: these materials are called thixotropic materials (see e.g. [22]). In could be relatively easy to use a metamaterial undergoing solid-solid phase transitions in the design of sound screen to stop undesired mechanical vibrations (the modeling of the phenomenon is presented e.g. in $[213,65]$. Damping effect needed to obtain energy adsorption from mechanical vibrations could consist in the induced phase transition, which in one direction should be endoenergetic.

Of course strong non-linearities and non-convexities could be involved in the description of such phenomena whose mathematical description could require the formulation of higher gradient or micro-structured continuum models.

Another interesting class of phenomena involving efficient and volume distributed dissipation effects are those dissipation phenomena occurring in porous deformable solids (see e.g. [167, 179]) possibly partially saturated (see e.g. [131]) with compressible fluids because of deformation induced (conceivably) viscous flow.

The fluid can be totally or partially trapped in the pores, whose tortuosity may be more or less relevant (see also $[179,175,133,132,56,57,76]$ ). The design of metamaterials with fluid inclusions, which maybe completely or partially interconnected, or also totally or partially isolated (with possibly energy dissipat- 
ing channel opening mechanisms) is really promising: the effects of creation frequency band gaps already described may be enhanced by the existence of Biot-type slow and fast waves.

\subsection{Conception of sound or noise screens}

Related to the subject of the previous subsection is the concept of 'noise screen' to be designed by exploiting the peculiarities of metamaterials.

Screens are usually bodies to be modeled by means of bidimensional continua. The theoretical analysis of bi-dimensional continua endowed with micro-structure has recently found a great impulse. We refer to the papers $[152,153,154]$ for interesting mathematical results in the subject of Cosserat shells and to $[87,88,89]$ for interesting results about micro-structured shells in which phase transition may occur.

These shells may be designed to carry a mass and be endowed with suitable properties in order to stop noise: some investigations generalizing those presented in $[175,174,163,132,133,61]$ seem ready to give interesting results. The idea is again that of 'trapping' vibration energy in the internal degrees of freedom of the system, which this time are those of considered micro-structured shells.

Another class of phenomena which needs to be considered are those occurring in microstructured shells in presence of pre-stresses (see e.g. [7, 8, 10, 12]) or those involving shells with phase transitions [85, 86, 87, 161].

The comparative analysis performed in [163] implies that the interfacial mechanical properties may have very interesting and effective effects on wave transmission and reflection: therefore one should expect that pre-stressed shells, possibly able to undergo phase transitions, may act as very efficient noise screens in a wide range of frequencies and amplitudes.

Also exploitable can be those dissipative contact phenomena involving impact (see e.g. [53, 16]) or those phenomena related to so called energetic boundaries (see e.g. [189, 9, 11, 190, 191, 139]).

A research investigation to be performed in close future involves the formulation of a boundary value problem for micro-structured three-dimensional continua involving boundaries which are endowed with one or more than one of the aforementioned surfacial micro-structures. The structure of these boundary conditions may induce:

$\circ$ a time delay in the release of energy which is arriving at the interface;

$\circ$ a dissipation concentrated at the interface;

- a considerable increase of reflected energy, also with time delays;

$\circ$ a surface trapping of incident energy with its release in different forms (which could have non-parasitic uses);

- the formation of surface waves, involving surface displacement, surface thickness variation, or more generally surface micro-structural oscillations.

Shells are surfaces always constituted by the same material particles while the involved phenomena are occurring. The difficulties dramatically increase when interfaces endowed with material properties but constituted by different material particles (see $[58,59,55]$ ) are involved.

Actually materials which may undergo phase transition, in presence of mechanical vibrations, may exhibit the onset of internal boundary layers $([105,213,161])$ whose localization is not known a priori: the free moving boundary problems which may arise are also very difficult to be solved, but may produce another effective method for controlling the energy transfer in metamaterials. 


\subsection{Towards the design of new piezoelectromechanical transducers}

Up to now piezoelectric transduction were based on the principle of transforming every kind of deformation into a suitable elongation and therefore to use, for actuation, PETs which respond electrically to elongations. This concept has been really successful for designing bending transducers, i.e. transducers which produce an electric signal when the structural member to which are mechanically connected is bent, or viceversa transducers which apply bending couples when subjected to a suitable electric signal.

Bimorph transducers configuration allows for the transformation of bending into elongation and therefore the configuration in which elongating PETs allow for a bending actuation.

When dealing with shear deformation this concept cannot be easily adapted.

In the papers $[62,66]$ a problem of piezoelasticity is solved which seems to allow for the concept of a truly shearble PET. In the cited papers an Almansi-type problem in piezoelasticity is solved, by assigning on the mantle of a Saint-Venant cylinder a polynomial (with respect to the abscissa along a directrix of the cylinder) electrical potential and by calculating the corresponding imposed shear deformation.

\subsection{Design of metamaterials self-limiting and self-detecting damage effects}

Damage in materials is always considered as a negative by-product of motion or external actions.

It has been discussed how this apparently always negative effect may be used to obtained some positive microscopically driven damping effects, at least until the progress of damage does not cause a permanent structural failure.

Healing is that process which occurs in living tissues to repair the effects of the progress of damage.

A great scientific and technological challenge is the conception of self-healing materials. More modestly one could imagine systems which are able to self-limit the effects of damage and to continue to be effective also in presence of serious damage effects.

To this category surely belong the already described piezoelectromechanical systems.

Indeed in an initial stage of crack formation in their mechanical part piezoelectromechanical structures may continue to resist to external loads if the transduction system is efficient enough to allow for the transformation of mechanical energy into its electrical counterpart.

It needs to be investigated the range of validity of aforementioned statement. In particular it has to be assessed

o when the extension of mechanical damage actually reduces the whole system to failure

- which degree of damage in the transduction system does not affect an effective transduction

o when the damage in the electrical part of the whole wave-guide renders useless the piezoelectromechanical transduction.

However the coupled electric and mechanical systems have a peculiarity which needs to be further explored: indeed it is possible to exploit the efficiency and sensitivity of electronic circuits to assess, with periodic electrically driven checks or even in presence of measured malfunctioning of the electric wave-guide, the eventual damage progress in the system.

Actually one can easily design a system of signaling and collection of signals in the electric network whose anomalies, when suitably compared with a series of results of numerical simulations, may allow for a self-detection of damage.

This concept could have a great impact in a large class of structural systems. 


\section{References}

[1] S. Alessandroni, F. dell'Isola, and M. Porfiri. A revival of electric analogs for vibrating mechanical systems aimed to their efficient control by PZT actuators. International Journal of Solids and Structures, $39(20), 2002,5295-5324$.

[2] S. Alessandroni, F. dell'Isola, and F. Frezza. Optimal piezo-electro-mechanical coupling to control plate vibrations. International Journal of Applied Electromagnetics and Mechanics, 13(1-4), 2002, 113-120.

[3] S. Alessandroni, U. Andreaus, and F. dell'Isola. A novel passive electric network analog to KirchhoffLove plate designed to efficiently damp forced vibrations by distributed piezoelectric transducers. Proceedings of SPIE - The International Society for Optical Engineering, W.K. Agnes G.S., San Diego, CA: $2003,380-391$.

[4] S. Alessandroni, U. Andreaus, F. dell'Isola, and M. Porfiri. Piezo-ElectroMechanical (PEM) Kirchhoff-Love plates. European Journal of Mechanics/A Solids, 23(4), 2004, 689-702.

[5] S. Alessandroni, U. Andreaus, F. dell'Isola, and M. Porfiri. A passive electric controller for multimodal vibrations of thin plates. Computers and Structures, 83(15-16), 2005, 1236-1250.

[6] Alibert, J.J., Seppecher, P., \& dell'Isola, F. (2003). Truss modular beams with deformation energy depending on higher displacement gradients. Mathematics and Mechanics of Solids, 8, 51-73.

[7] Altenbach, H., \& Eremeyev, V.A. (2009). Eigen-vibrations of plates made of functionally graded material. Computers, Materials and Continua, 9(2), 153-177.

[8] Altenbach, H., Eremeyev, V.A., \& Morozov, N.F. (2010). On equations of the linear theory of shells with surface stresses taken into account. Mechanics of Solids, 45(3), 331-342.

[9] Altenbach, H., Eremeyev, V.A., \& Lebedev, L.P. (2010). On the existence of solution in the linear elasticity with surface stresses. ZAMM Zeitschrift für Angewandte Mathematik und Mechanik, 90(3), 231-240.

[10] Altenbach, H., \& Eremeyev, V.A. (2010). On the effective stiffness of plates made of hyperelastic materials with initial stresses. International Journal of Non-Linear Mechanics, 45(10), 976-981.

[11] Altenbach, H., \& Eremeyev, V.A. (2011). On the shell theory on the nanoscale with surface stresses. International Journal of Engineering Science, 49(12), 1294-1301.

[12] Altenbach, H., Eremeyev, V.A., \& Morozov, N.F. (2012). Surface viscoelasticity and effective properties of thin-walled structures at the nanoscale. International Journal of Engineering Science, 59 83-89.

[13] U. Andreaus, F. dell'Isola, and M. Porfiri. Piezoelectric passive distributed controllers for beam flexural vibrations, 14th U.S. National Congress of Applied Mechanics, Blacksburg, VA: 2002.

[14] U. Andreaus, F. dell'Isola, and M. Porfiri. Piezoelectric Passive Distributed Controllers for Beam Flexural Vibrations, Journal of Vibration and Control, 10(5), 2004, 625-659.

[15] Andreaus, U. \& Baragatti, P. (2009). Fatigue crack growth, free vibrations, and breathing crack detection of aluminium alloy and steel beams. Journal of Strain Analysis for Engineering Design, 44(7), 595-608. 
[16] Andreaus, U., Placidi, L., \& Rega, G. (2010). Numerical simulation of the soft contact dynamics of an impacting bilinear oscillator. Communications in Nonlinear Science and Numerical Simulation, 15(9), 2603-2616.

[17] U. Andreaus, I. Giorgio, and A. Madeo. Modeling of the interaction between bone tissue and resorbable biomaterial as linear elastic materials with voids. ZAMP - Zeitschrift für Angewandte Mathematik und Physik / Journal of Applied Mathematics and Physics, pages 1-29, 2014.

[18] ANSI/IEEE Std 176-1987. IEEE standard on piezoelectricity. The Institute of Electrical and Electronics Engineers, Inc, 1987.

[19] Auffray, N., Bouchet, R., \& Brechet, Y. (2010). Strain gradient elastic homogenization of bidimensional cellular media. International Journal of Solids and Structures, 47(13), 1698-1710.

[20] T. Bailey and J. E. Ubbard. Distributed piezoelectric-polymer active vibration control of a cantilever beam, Journal of Guidance, Control, and Dynamics, 8(5) (1985), 605-611. doi: 10.2514/3.20029

[21] T. Bailey, T., A. Gruzen, and P. Madden. RCS/piezoelectric distributed actuator study. No. CSDL-R2076. Charles Stark Draper Laboratory, Inc. Cambridge, MA 1988.

[22] Barnes., H. A.(1997). Thixotropy - a review. Journal of Non-Newtonian Fluid Mechanics, 70(1-2), 1-33. http://dx.doi.org/10.1016/S0377-0257(97)00004-9.

[23] R. Batra, F. dell'Isola, S. Vidoli, and D. Vigilante. Multimode vibration suppression with passive twoterminal distributed network incorporating piezoceramic transducers. International Journal of Solids and Structures, 42(11-12), 2005, 3115-3132.

[24] S. Behrens, S. O. R. Moheimani, and A. J. Fleming. Multiple mode current flowing passive piezoelectric shunt controller. Journal of Sound and Vibration, 266:929-942, 2003.

[25] Lim, Y. Y., S. Bhalla, et al. (2006). Structural identification and damage diagnosis using self-sensing piezo-impedance transducers. Smart Materials And Structures 15: 987-995.

[26] Bhalla, Suresh, and Chee Kiong Soh. Piezo-Impedance Transducers for Evaluation of Seismic Induced Structural Damage. Earthquakes and Health Monitoring of Civil Structures. Springer Netherlands, 2013, 133-148.

[27] J.L. Bleustein, A note on the boundary conditions of Toupin's strain gradient-theory, Int. J. Solids Structures 3 (1967) 1053-1057.

[28] L.-T. Bruton, RC-Active Circuits: Theory and Design, Prentice-Hall, 1980.

[29] Carcaterra, A., Akay, A. Transient energy exchange between a primary structure and a set of oscillators: Return time and apparent damping (2004) Journal of the Acoustical Society of America, 115(2), 683696.

[30] Koç, I.M., Carcaterra, A., Xu, Z., Akay, A. Energy sinks: Vibration absorption by an optimal set of undamped oscillators (2005) Journal of the Acoustical Society of America, 118(5), 3031-3042.

[31] Carcaterra, A. Ensemble energy average and energy flow relationships for nonstationary vibrating systems (2005) Journal of Sound and Vibration, 288 (3), 751-790. 
[32] Carcaterra, A., Akay, A., Koç, I.M. Near-irreversibility in a conservative linear structure with singularity points in its modal density (2006) Journal of the Acoustical Society of America, 119(4), 2141-2149.

[33] Carcaterra, A., Akay, A. Theoretical foundations of apparent-damping phenomena and nearly irreversible energy exchange in linear conservative systems (2007) Journal of the Acoustical Society of America, 121(4), 1971-1982.

[34] Carcaterra, A., Akay, A., Lenti, F. Pseudo-damping in undamped plates and shells (2007) Journal of the Acoustical Society of America, 122(2), 804-813.

[35] A. Carcaterra, A. Akay, Dissipation in a finite-size bath, Physical Review E 84 (2011).

[36] A. Carcaterra, A. Sestieri, Energy Density Equations and Power Flow in Structures, Journal of Sound and Vibration 188 (1995) 269-282.

[37] A. Carcaterra, E. Ciappi, A. Iafrati, E.F. Campana, Shock spectral analysis of elastic systems impacting on the water surface, Journal of Sound and Vibration 229 (2000) 579-605.

[38] Antonio Carcaterra, Daniele Dessi, Franco Mastroddi, Hydrofoil vibration induced by a random flow: a stochastic perturbation approach, Journal of Sound and Vibration 283 (1-2), 6 May 2005, 401-432, http://dx.doi.org/10.1016/j.jsv.2004.04.040.

[39] Cazzani, A. On the dynamics of a beam partially supported by an elastic foundation: An exact solutionset (2013) International Journal of Structural Stability and Dynamics, 13(8), art. no. 1350045.

[40] Cazzani, A., Ruge, P. Numerical aspects of coupling strongly frequency-dependent soil-foundation models with structural finite elements in the time-domain (2012) Soil Dynamics and Earthquake Engineering, 37, 56-72.

[41] Cazzani, A., Lovadina, C. On some mixed finite element methods for plane membrane problems (1997) Computational Mechanics, 20(6), 560-572.

[42] Cazzani, A., Atluri, S.N. Four-noded mixed finite elements, using unsymmetric stresses, for linear analysis of membranes (1993) Computational Mechanics, 11(4), 229-251.

[43] Chopra I. Review of state of art of smart structures and integrated systems. AIAA Journal 2002; 40(11): $2145-2187$.

[44] Chung SL, Leung KS, Cheung WH. Low-magnitude high-frequency vibration enhances gene expression related to callus formation, mineralization and remodeling during osteoporotic fracture healing in rats. J Orthop Res. 2014 Aug 17

[45] Clark W., Vibration control with states-switched piezoelectric materials. J. of Intelligent Mat. Sys. And Struct. 11, 263-273 (2000).

[46] Coor L.R., Clark W.W., A novel semi-active multimodal vibration control law for a piezoceramic actuator. J. Vibration and Acoustics 125, 214-222 (2003).

[47] L. Contrafatto, M. Cuomo, A globally convergent numerical algorithm for damaging elasto-plasticity based on the Multiplier method, International Journal for Numerical Methods in Engineering 63 (2005) $1089-1125$. 
[48] L. Contrafatto, M. Cuomo, A new thermodynamically consistent continuum model for hardening plasticity coupled with damage, International Journal of Solids and Structures 39 (2002) 6241-6271.

[49] L. Contrafatto, M. Cuomo, A framework of elastic-plastic damaging model for concrete under multiaxial stress states, International Journal of Plasticity 22 (2006) 2272-2300.

[50] Crandall, S.H., Karnopp, D.C., Kurtz Jr., E.F., Pridmore-Brown, F.C., 1968. Dynamics of Mechanical and Electromechanical Systems. McGraw- Hill, New York.

[51] A. Culla, A. Sestieri, A. Carcaterra, Energy flow uncertainties in vibrating systems: Definition of a statistical confidence factor, Mechanical Systems and Signal Processing 17 (2003) 635-663.

[52] Cuomo, M., Greco, L. Isogeometric analysis of space rods: Considerations on stress locking (2012) ECCOMAS 2012 - European Congress on Computational Methods in Applied Sciences and Engineering, e-Book Full Papers, 5094-5112.

[53] M. Cuomo, G. Ventura, Complementary Energy Approach to Contact Problems Based on Consistent Augmented Lagrangian regularization, Mathematical and Computer Modelling 28 (1998) 185-204.

[54] M. Cuomo, L. Contrafatto, Stress rate formulation for elastoplastic models with internal variables based on augmented Lagrangian regularisation, International Journal of Solids and Structures, 37 (2000) 3935-3964.

[55] F. dell'Isola, A. Romano, A phenomenological approach to phase transition in classical field theory, International Journal of Engineering Science, 25 (1987) 1469-1475.

[56] F. dell'Isola, L. Rosa, L., C. Wozniak, Dynamics of solids with micro periodic nonconnected fluid inclusions, Archive of Applied Mechanics 67(4) (1997) 215-228.

[57] F. dell'Isola, L. Rosa, L., C. Wozniak, A micro-structured continuum modelling compacting fluidsaturated grounds: The effects of pore-size scale parameter, Acta Mechanica 127(1-4) (1998) 165-182.

[58] F. dell'Isola, A. Romano, On a general balance law for continua with an interface, Ricerche di Matematica, 35 (1986) 325-337.

[59] F. dell'Isola, A. Romano, On the derivation of thermomechanical balance equations for continuous systems with a nonmaterial interface, International Journal of Engineering Science, 25 (1987) 14591468.

[60] F. dell'Isola, A. Madeo, P. Seppecher, Boundary conditions at fluid-permeable interfaces in porous media: A variational approach, International Journal of Solids and Structures 46 (17) (2009) 31503164 .

[61] dell'Isola F., Madeo A., Placidi L., 2012. Linear plane wave propagation and normal transmission and reflection at discontinuity surfaces in second gradient 3D Continua. Zeitschrift für Angewandte Mathematik und Mechanik (ZAMM), 92:1, 52-71

[62] F. dell'Isola and L. Rosa. St. Venant problem in linear piezoelectricity. Proceedings of SPIE - The International Society for Optical Engineering, C.J. Varadan Vasundara V., San Diego, CA, USA: 1996, 399-409. 
[63] F. dell'Isola and S. Vidoli. Distributed control of beams by electric transmission lines with PZT actuators. Proceedings of SPIE - The International Society for Optical Engineering, Adelaide, SA: 1997, $312-321$.

[64] F. dell'Isola, C. Woźniak, On phase transition layers in certain micro-damaged two-phase solids, International Journal of Fracture 83(2) (1997) 175-189.

[65] F. dell'Isola, C. Woźniak, On continuum modelling the interphase layers in certain two-phase elastic solids, ZAMM Zeitschrift für Angewandte Mathematik und Mechanik 77(7) (1997) 519-526.

[66] F. dell'Isola and L. Rosa. Almansi-type boundary conditions for electric potential inducing flexure in linear piezoelectric beams. Continuum Mechanics and Thermodynamics, 9(2), 1997, 115-125.

[67] F. dell'Isola and S. Vidoli. Damping of bending waves in truss beams by electrical transmission lines with PZT actuators. Archive of Applied Mechanics, 68(9), 1998, 626-636.

[68] F. dell'Isola and S. Vidoli. Continuum modelling of piezoelectromechanical truss beams: An application to vibration damping. Archive of Applied Mechanics, 68(1), 1998, 1-19.

[69] F. dell'Isola, E.G. Henneke, and M. Porfiri. Synthesis of electrical networks interconnecting PZT actuators to damp mechanical vibrations. International Symposium on Applied Electromagnetics and Mechanics in honor of Professor K.Miya, Tokyo: 2000.

[70] F. dell'Isola, E.G. Henneke, and M. Porfiri. Synthesis of electrical networks interconnecting PZT actuators to damp mechanical vibrations. International Journal of Applied Electromagnetics and Mechanics, 14(1-4), 2002, 417-424.

[71] F. dell'Isola, F. Vestroni, and S. Vidoli. A class of electro-mechanical systems: linear and nonlinear dynamics. Journal of Theoretical and Applied Mechanics, 40(1), 2002, 47-71.

[72] F. dell'Isola, M. Porfiri, and S. Vidoli. Piezo-electromechanical (PEM) structures: Passive vibration control using distributed piezoelectric transducers. Comptes Rendus - Mecanique, 331(1), 2003, 69-76.

[73] F. dell'Isola, E. Santini, and D. Vigilante. Purely electrical damping of vibrations in arbitrary PEM plates: A mixed non-conforming FEM-Runge-Kutta time evolution analysis. Archive of Applied Mechanics, 73(1-2), 2003, 26-48.

[74] F. dell'Isola, C. Maurini, and M. Porfiri. Passive damping of beam vibrations through distributed electric networks and piezoelectric transducers: Prototype design and experimental validation. Smart Materials and Structures, 13(2), 2004, 299-308.

[75] F. dell'Isola, F. Vestroni, and S. Vidoli. Structural-damage detection by distributed piezoelectric transducers and tuned electric circuits. Research in Nondestructive Evaluation, 16, 2005, 101-118.

[76] F. dell'Isola, K. Hutter. What are the dominant thermomechanical processes in the basal sediment layer of large ice sheets? Proceedings of the Royal Society of London. Series A: Mathematical, Physical and Engineering Sciences 454 (1998) 1169-1195.

[77] F. dell'Isola, P. Seppecher, The relationship between edge contact forces, double force and interstitial working allowed by the principle of virtual power, Comptes rendus de l'Académie des Sciences Serie IIb, 321 (1995) 303-308. 
[78] F. dell'Isola, P. Seppecher, A. Madeo, How contact interactions may depend on the shape of Cauchy cuts in N-th gradient continua: approach à la D'Alembert, Zeitschrift für Angewandte Mathematik und Physik (ZAMP) 63 (2012) 1119-1141.

[79] F. dell'Isola, U. Andreaus, and L. Placidi. At the origins and in the vanguard of peridynamics, non-local and higher-gradient continuum mechanics: An underestimated and still topical contribution of Gabrio Piola Mathematics and Mechanics of Solids 1081286513509811, first published on February 2, 2014 as doi:10.1177/1081286513509811

[80] dell'Isola, F., and Steigmann, D. A Two-Dimensional Gradient-Elasticity Theory for Woven Fabrics. Journal of Elasticity (2014): 1-13.

[81] Di Egidio, A., Luongo, A., Paolone, A. Linear and non-linear interactions between static and dynamic bifurcations of damped planar beams (2007) International Journal of Non-Linear Mechanics, 42(1), 88-98.

[82] Dimitriadis, E. K., C. R. Fuller, and C. A. Rogers. Piezoelectric actuators for distributed vibration excitation of thin plates. Journal of Vibration and Acoustics 113.1 (1991): 100-107.

[83] Engheta Nader, Richard W. Ziolkowski, Metamaterials: Physics and Engineering Explorations, Wiley \& Sons 2006.

[84] V.A. Eremeyev, A.B. Freidin, L.L., Sharipova, Nonuniqueness and Stability in Problems of Equilibrium of Elastic Two-Phase Bodies, Doklady Physics 48(7) (2003) 359-363.

[85] V.A. Eremeyev, W. Pietraszkiewicz, The nonlinear theory of elastic shells with phase transitions, Journal of Elasticity 74(1) (2004) 67-86.

[86] V.A. Eremeyev, W. Pietraszkiewicz, Local symmetry group in the general theory of elastic shells, Journal of Elasticity 85(2) (2006) 125-152.

[87] V.A. Eremeyev, L.M. Zubov, On constitutive inequalities in nonlinear theory of elastic shells, ZAMM Zeitschrift für Angewandte Mathematik und Mechanik 87(2) (2007) 94-101.

[88] V.A. Eremeyev, H. Altenbach, N.F. Morozov, The influence of surface tension on the effective stiffness of nanosize plates, Doklady Physics 54(2) (2009) 98-100.

[89] V.A. Eremeyev, W. Pietraszkiewicz, Thermomechanics of shells undergoing phase transition, Journal of the Mechanics and Physics of Solids 59 (2011) 1395-1412.

[90] A.C. Eringen, E.S. Suhubi, Nonlinear theory of simple microelastic solids: I, Int. J. Eng. Sci. 2 (1964) 189-203.

[91] A.C. Eringen, E.S. Suhubi, Nonlinear theory of simple microelastic solids: II, Int. J. Eng. Sci. 2 (1964) 389-404.

[92] A.C. Eringen, D.G.B. Edelen, On nonlocal Elasticity, Int. J. Eng. Sci. 10 (1972) 233-248.

[93] A. C. Eringen, Microcontinuum Field Theories I. Foundations and Solids, Springer Verlag (1999).

[94] A.C. Eringen, Microcontinuum field theories, Springer-Verlag, New York, 2001. 
[95] A.Cemal Eringen, Nonlocal continuum field theories, Springer (2002).

[96] Erturk, Alper, and Daniel J. Inman. Piezoelectric energy harvesting. John Wiley \& Sons, 2011.

[97] Erturk, Alper, and Daniel J. Inman. Issues in mathematical modeling of piezoelectric energy harvesters. Smart Materials and Structures 17.6 (2008): 065016.

[98] Erturk, Alper, and Daniel J. Inman. A distributed parameter electromechanical model for cantilevered piezoelectric energy harvesters. Journal of Vibration and Acoustics 130.4 (2008): 041002.

[99] Farrar, C. R., S. W. Doebling, et al. (2001). Vibration-based structural damage identification. Philosophical Transactions: Mathematical, Physical and Engineering Sciences: 359 (1778): 131-149.

[100] S. Federico, W. Herzog, Towards an analytical model of soft biological tissues, Journal of Biomechanics, 41(16) (2008) 3309-3313.

[101] S. Federico, A. Grillo, W. Herzog, A transversely isotropic composite with a statistical distribution of spheroidal inclusions: A geometrical approach to overall properties, Journal of the Mechanics and Physics of Solids 52(10) (2004) 2309-2327.

[102] S. Federico, T.C. Gasser, Nonlinear elasticity of biological tissues with statistical fibre orientation, Journal of the Royal Society Interface 7(47) (2010) 955-966.

[103] S. Federico, A. Grillo, G. La Rosa, G. Giaquinta, W. Herzog, A Transversely Isotropic, Transversely Homogeneous Microstructural-Statistical Model of Articular Cartilage, JBIOM 38 (2005) 2008-2018.

[104] S. Federico and A. Grillo, Elasticity and Permeability of Porous Fibre-Reinforced Materials Under Large Deformations, Mech. Mat. 44 (2012) 58-71.

[105] Ferretti, M. Ferretti, A. Madeo, F. dell'Isola, P. Boisse, Modeling the onset of shear boundary layers in fibrous composite reinforcements by second-gradient theory. Zeitschrift für angewandte Mathematik und Physik (2013): 1-26.

[106] S. Forest, R. Sievert, Nonlinear microstrain theories, Int. J. Solids Struct. 43 (2006) 7224-7245.

[107] S. Forest, Micromorphic Approach for Gradient Elasticity, Viscoplasticity, and Damage, Journal of Engineering Mechanics 135(3) (2009) 117-131.

[108] R.-L. Forward, G.-J. Swigert, Electronic damping of orthogonal bending modes in a cylindrical masttheory, J. Spacecraft Rockets 18 (1981) 5-10.

[109] R. Gatignol, P. Seppecher, Modelisation of fluid-fluid interfaces with material properties, Journal de Mécanique Théorique et Appliquée (1986) 225-247.

[110] I. Giorgio, A. Culla, D. Del Vescovo, Multimode vibration control using several piezoelectric transducers shunted with a multiterminal network, Archive of Applied Mechanics 79(9) (2009) 859-879.

[111] I.D. Ghiba, P. Neff, A. Madeo, L. Placidi, G. Rosi. The relaxed linear micromorphic continuum: existence, uniqueness and continuous dependence in dynamics. Mathematics and Mechanics of Solids (2014): 1081286513516972.

[112] Greco, L., Cuomo, M. B-Spline interpolation of Kirchhoff-Love space rods (2013) Computer Methods in Applied Mechanics and Engineering, 256, 251-269. 
[113] Greco, L., Cuomo, M. On the force density method for slack cable nets (2012) International Journal of Solids and Structures, 49(13), 1526-1540.

[114] Guyomard D., Badel A., Nonlinear semi-passive multimodal vibration damping : A efficient probalistic approach. J. Sound and Vibration 294, 249-268 (2006).

[115] N.-W. Hagood, A. von Flotow, Damping of structural vibrations with piezoelectric materials and passive electrical networks, J. Sound Vib. 146 (1991) 243-268.

[116] Halim, D. and S. O. R. Moheimani (2003). An optimization approach to optimal placement of collocated piezoelectric actuators and sensors on a thin plate. Mechatronics 8, 27-47.

[117] J.-P. den Hartog, Mechanical Vibrations, McGraw-Hill, New York, 1934.

[118] J. J. Hollkamp. Multimodal passive vibration suppression with piezoelectric materials and resonant shunts. Journal of Intelligent Material Systems and Structures, 5:49-57, 1994.

[119] E. Kroner, Mechanics of Generalized Continua, Springer (1968).

[120] Lallart M., Le Feuvre E., Ricgard C., Guyomard D., Self powered circuit for broadband multimodal piezoelectric vibration control. Sensors and Actuators A 143, 377-382 (2008).

[121] Lee, C. K. Theory of laminated piezoelectric plates for the design of distributed sensors/actuators. Part I: Governing equations and reciprocal relationships. The Journal of the Acoustical Society of America 87.3 (1990): 1144-1158.

[122] T. Lekszycki, F. dell'Isola, A mixture model with evolving mass densities for describing synthesis and resorption phenomena in bones reconstructed with bio-resorbable materials, ZAMM - Journal of Applied Mathematics and Mechanics / Zeitschrift für Angewandte Mathematik und Mechanik (2012).

[123] A. Luongo, A. Di Egidio, Bifurcation equations through multiple-scales analysis for a continuous model of a planar beam, Nonlinear Dynamics 41 (2005) 171-190.

[124] Luongo, A., Piccardo, G. Linear instability mechanisms for coupled translational galloping (2005) Journal of Sound and Vibration, 288(4-5), 1027-1047.

[125] Luongo, A., Paolone, A. Perturbation Methods for Bifurcation Analysis from Multiple Nonresonant Complex Eigenvalues (1997) Nonlinear Dynamics, 14 (3), 193-210.

[126] Luongo, A., Zulli, D., Piccardo, G. On the effect of twist angle on nonlinear galloping of suspended cables (2009) Computers and Structures, 87(15-16), 1003-1014.

[127] Luongo, A., Zulli, D., Piccardo, G. A linear curved-beam model for the analysis of galloping in suspended cables (2007) Journal of Mechanics of Materials and Structures, 2 (4), 675-694.

[128] Luongo, A., Paolone, A., Piccardo, G. Postcritical Behavior of Cables Undergoing Two Simultaneous Galloping Modes (1998) Meccanica, 33(3), 229-242.

[129] A. Madeo, T. Lekszycki, F. dell'Isola, A continuum model for the bio-mechanical interactions between living tissue and bio-resorbable graft after bone reconstructive surgery, Comptes Rendus - Mecanique 339(10) (2011) 625-640. 
[130] A. Madeo, D. George, T. Lekszycki, M. Nierenberger, Y. Rémond, A second gradient continuum model accounting for some effects of micro-structure on reconstructed bone remodelling, Comptes Rendus Mécanique 340(8) (2012) 575-589.

[131] A. Madeo, F. dell'Isola, F. Darve, A continuum model for deformable, second gradient porous media partially saturated with compressible fluids, Journal of the Mechanics and Physics of Solids 61 (2013) $2196-2211$.

[132] A. Madeo, S. Gavrilyuk, Propagation of acoustic waves in porous media and their reflection and transmission at a pure-fluid/porous-medium permeable interface European, Journal of Mechanics-A/Solids 29(5) (2010) 897-910.

[133] A. Madeo, I. Djeran-Maigre, G. Rosi, C. Silvani, The effect of fluid streams in porous media on acoustic compression wave propagation, transmission, and reflection, Continuum Mechanics and Thermodynamics 25 (2013) 173-196.

[134] K. Manktelow, M.J. Leamy, M. Ruzzene, Topology Design and Optimization of Nonlinear Periodic Materials, Journal of the Mechanics and Physics of Solids (2013) in press.

[135] G.A. Maugin, A.V. Metrikine (eds.) Mechanics of generalized Continua One hundred years after the Cosserats, Springer (2010).

[136] C. Maurini, J. Pouget, and F. dell'Isola. On a model of layered piezoelectric beams including transverse stress effect. International journal of solids and structures, 41 (16-17), 2004, 4473-4502.

[137] C. Maurini, F. dell'Isola, and D. Del Vescovo. Comparison of piezoelectronic networks acting as distributed vibration absorbers. Mechanical Systems and Signal Processing, 18(5), 2004, 1243-1271.

[138] C. Maurini, J. Pouget, and F. dell'Isola. Extension of the Euler Bernoulli model of piezoelectric laminates to include 3D effects via a mixed approach. Computers and Structures, 84 (22-23), 2006, 14381458 .

[139] A.T. McBride, A. Javili, P. Steinmann, S. Bargmann, Geometrically nonlinear continuum thermomechanics with surface energies coupled to diffusion, Journal of the Mechanics and Physics of Solids 59 (2011) 2116-2133.

[140] A.T. McBride, J. Mergheim, A. Javili, P. Steinmann, S. Bargmann, Micro-to-macro transitions for heterogeneous material layers accounting for in-plane stretch, Journal of the Mechanics and Physics of Solids 60 (2012) 1221-1239.

[141] R.D. Mindlin, Micro-structure in linear elasticity, Archive for Rational Mechanics and Analysis 16 (1964) 51-78.

[142] A. Misra, V. Singh, Micromechanical model for viscoelastic-materials undergoing damage, Continuum Mechanics and Thermodynamics, 25 (2013) 1-16.

[143] A. Misra, W.Y. Ching, Theoretical nonlinear response of complex single crystal under multi-axial tensile loading, Scientific Reports, 3 (2013).

[144] S. O. R. Moheimani. A survey of recent innovezions in vibration damping and control using shuntend piezoelectric transducers. IEEE Transactions on Control Systems Technology, 11(4):482-494, 2003. 
[145] J. N. Juang and M. Phan. Robust controller design for second-order dynamic systems: a virtual passive approach. Journal of Guidance, Control and Dynamics, 15:1192-1198, 1992.

[146] B. Nadler, P. Papadopoulos, D.J. Steigmann, Multiscale constitutive modeling and numerical simulation of fabric material, International Journal of Solids and Structures, 43 (2006) 206-221.

[147] R.K. Narisetti, M. Ruzzene, M.J. Leamy, Study of Wave Propagation in Strongly Nonlinear Periodic Lattices Using a Harmonic Balance Approach, Wave Motion, 49 (2012) 394-410.

[148] R.K. Narisetti, M. Ruzzene, M.J. Leamy, A Perturbation Approach for Analyzing Dispersion and Group Velocities in Two-Dimensional Nonlinear Periodic Lattices, Journal of Vibration and Acoustics 133(6) 2011 1-12.

[149] P. Neff, I.D. Ghiba, A. Madeo, L. Placidi, G. Rosi, A unifying perspective: the relaxed linear micromorphic continuum, Continuum Mechanics and Thermodynamics 26(5) 2014 639-681.

[150] P. Neff, On Korn's first inequality with non-constant coefficients, Royal Society of Edinburgh - Proceedings A 132 (1) (2002) 221-243.

[151] P. Neff, J. Jeong, A new paradigm: The linear isotropic Cosserat model with conformally invariant curvature energy, ZAMM Zeitschrift für Angewandte Mathematik und Mechanik 89 (2) (2009) 107-122.

[152] P. Neff, A geometrically exact planar cosserat shell-model with microstructure: Existence of minimizers for zero cosserat couple modulus, Mathematical Models and Methods in Applied Sciences 17(3) (2007) 363-392.

[153] P. Neff, A geometrically exact Cosserat shell-model including size effects, avoiding degeneracy in the thin shell limit. Part I: Formal dimensional reduction for elastic plates and existence of minimizers for positive Cosserat couple modulus, Continuum Mechanics and Thermodynamics 16(6) (2004) 577-628.

[154] P. Neff, K. CheŁmiński, A geometrically exact Cosserat shell model for defective elastic crystals Justification via-convergence, Interfaces and Free Boundaries 9(4) (2007) 455-492.

[155] Oliveto, G., Cuomo, M. Incremental analysis of plane frames with geometric and material nonlinearities (1988) Engineering Structures, 10(1), 2-12.

[156] H.-F. Olsen, Electronic control of noise, vibration and reverberation, J. Acoust. Soc. 28 (1956) 972-976.

[157] Paolone, A., Vasta, M., Luongo, A. Flexural-torsional bifurcations of a cantilever beam under potential and circulatory forces I: Non-linear model and stability analysis (2006) International Journal of NonLinear Mechanics, 41(4), 586-594.

[158] Park, K.-I., Son, J. H., Hwang, G.-T., Jeong, C. K., Ryu, J., Koo, M., Choi, I., Lee, S. H., Byun, M., Wang, Z. L. and Lee, K. J. (2014), Highly-Efficient, Flexible Piezoelectric PZT Thin Film Nanogenerator on Plastic Substrates. Adv. Mater., 26: 2514-2520. doi: 10.1002/adma.201305659

[159] Piccardo, G. A methodology for the study of coupled aeroelastic phenomena (1993) Journal of Wind Engineering and Industrial Aerodynamics, 48(2-3), 241-252.

[160] Piccardo, G., Tubino, F. Simplified procedures for vibration serviceability analysis of footbridges subjected to realistic walking loads (2009) Computers and Structures, 87(13-14), 890-903. 
[161] W. Pietraszkiewicz, V.A. Eremeyev, V. Konopinska, Extended non-linear relations of elastic shells undergoing phase transitions, Zeitschrift für Angewandte Mathematik und Mechanik (ZAMM) 87 (2007) $150-159$.

[162] S. Pietrzko and Q. Mao. New results in active and passive control of sound transmission through double wall structures. Aerospace Science and Technology, 12:42-53, 2008.

[163] L. Placidi, G. Rosi, I. Giorgio, A. Madeo, Reflection and transmission of plane waves at surfaces carrying material properties and embedded in second-gradient materials, Mathematics and Mechanics of Solids (2013) doi: 10.1177/1081286512474016.

[164] M. Porfiri and F. dell'Isola. Multimodal beam vibration damping exploiting PZT transducers and passive distributed circuits. Journal de Physique IV France, 115(1), 2004, 323-330.

[165] M. Porfiri, F. dell'Isola, and F. M. Frattale Mascioli. Circuit analog of a beam and its application to multimodal vibration damping, using piezoelectric transducers. International Journal of Circuit Theory and Applications, 32(4), 2004, 167-198.

[166] M. Porfiri, F. dell'Isola, and E. Santini. Modeling and design of passive electric networks interconnecting piezoelectric transducers for distributed vibration control. International Journal of Applied Electromagnetics and Mechanics, 21(2), 2005, 69-87.

[167] S. Quiligotti, G.A. Maugin, F. dell'Isola, An Eshelbian approach to the nonlinear mechanics of constrained solid-fluid mixtures, Acta Mechanica 160 (2003) 45-60.

[168] Qiu, Jinhao, and Junji Tani. Vibration control of a cylindrical shell using distributed piezoelectric sensors and actuators. Journal of Intelligent Material Systems and Structures 6.4 (1995): 474-481.

[169] J.A. Mitchell, J.N. Reddy (1995) A refined hybrid plate theory for composite laminates with piezoelectric laminae. International Journal of Solids and Structures, 32(16), 2345-2367.

[170] A. Rinaldi, Y.C. Lai, Statistical damage theory of 2D lattices: Energetics and physical foundations of damage parameter, International Journal of Plasticity 23 (2007) 1796-1825.

[171] A. Rinaldi, A rational model for 2D Disordered Lattices Under Uniaxial Loading, Int. J. Damage Mech. 18 (2009) 233-57.

[172] A. Rinaldi, Bottom-up modeling of damage in heterogeneous quasi-brittle solids, Continuum Mechanics and Thermodynamics 25(2-4) (2013) 359-373.

[173] Romeo, F., Luongo, A. Vibration reduction in piecewise bi-coupled periodic structures (2003) Journal of Sound and Vibration, 268(3), 601-615.

[174] G. Rosi, I. Giorgio, and V. A. Eremeyev. Propagation of linear compression waves through plane interfacial layers and mass adsorption in second gradient fluids. ZAMM - Journal of Applied Mathematics and Mechanics/Zeitschrift für Angewandte Mathematik und Mechanik 93.12 (2013): 914-927.

[175] G. Rosi, A. Madeo, J.L. Guyader, Switch between fast and slow Biot compression waves induced by "second gradient microstructure at material discontinuity surfaces in porous media, International Journal of Solids and Structures 50(10) (2013) 1721-1746. 
[176] G. Rosi, J. Pouget and F. dell'Isola, "Control of sound radiation and transmission by a piezoelectric plate with an optimized resistive electrode", European Journal of Mechanics, A/Solids, 29(5), 2010, 859-870.

[177] Roveri, N., Carcaterra, A., Akay, A. Energy equipartition and frequency distribution in complex attachments (2009) Journal of the Acoustical Society of America, 126(1), 122-128.

[178] Sampaio R.P.C, Maia, N.M.M., Silvia, J.M.M, et al. (1999). Damage detection using the frequencyresponse-function curvature method. Journal of Sound and Vibration, 226(5) 1029-1042.

[179] G. Sciarra, F. dell'Isola, K. Hutter. A solid-fluid mixture model allowing for solid dilatation under external pressure. Continuum Mechanics and Thermodynamics, 13(5) (2001) 287-306.

[180] G. Sciarra, F. dell'Isola, O. Coussy, Second gradient poromechanics, Int. J. Solids Struct 44(20) (2007) $6607-6629$.

[181] L.I. Sedov, Models of continuous media with internal degrees of freedom, Journal of Applied Mathematics and Mechanics 32 (1972) 803-819.

[182] L.I. Sedov, Variational Methods of constructing Models of Continuous Media, Irreversible Aspects of Continuum Mechanics and Transfer of Physical Characteristics in Moving Fluids, Springer Vienna (1968) 346-358.

[183] P. Seppecher, Etude d'une Modelisation des Zones Capillaires Fluides: Interfaces et Lignes de Contact, Thése de l'Université Paris VI, Avril (1987).

[184] P. Seppecher, Thermodynamique des zones capillaires, Annales de Physique 13 (1988) 13-22.

[185] P. Seppecher, Etude des conditions aux limites en théorie du second gradient: cas de la capillarité, Comptes rendus de l'Académie des Sciences 309 (1989) 497-502.

[186] P. Seppecher, Equilibrium of a Cahn and Hilliard fluid on a wall: Influence of the wetting properties of the fluid upon the stability of a thin liquid film, European Journal of mechanics B/fluids 12 (1993) 69-84.

[187] P. Seppecher, Line Tension Effect upon Static Wetting, Oil and Gas Science and Technology- Rev. IFP, 56 (2001) $77-81$.

[188] H. Shen, J. Qiu, H. Ji, K. Zhu, M. Balsi, I. Giorgio and F. dell'Isola. A low-power circuit for piezoelectric vibration control by synchronized switching on voltage source. Sensors and Actuators A: Physical, 161(1-2), 2010, 245-255.

[189] P. Steinmann, On Boundary Potential Energies in Deformational and Configurational Mechanics, J. Mech. Phys. Solids Nr. 56, S. (2008) 772-80.

[190] H. Steeb, S. Diebels, Modeling thin films applying an extended continuum theory based on a scalarvalued order parameter - Part I: Isothermal case, International Journal of Solids and Structures 41 (2004) 5071-5085.

[191] D.J. Steigmann, R.W. Ogden, Plane deformations of elastic solids with intrinsic boundary elasticity, Proceedings of the Royal Society A: Mathematical, Physical and Engineering Sciences 453 (1997) 853877. 
[192] P. Steinmann, A. Elizondo, R. Sunyk, Studies of validity of the Cauchy-Born rule by direct comparison of continuum and atomistic modelling, Modelling and Simulation in Materials Science and Engineering 15 (2007).

[193] R. Sunyk, P. Steinmann, On Higher Gradients in Continuum-Atomistic Modelling, Int. J. Solids Structures 40 (2003) Nr. 24, S. 6877-6896.

[194] Sun, Dongchang, and Liyong Tong. Modal control of smart shells by optimized discretely distributed piezoelectric transducers. International Journal of Solids and Structures 38.18 (2001): 3281-3299.

[195] Swallow, L. M., et al. A piezoelectric fibre composite based energy harvesting device for potential wearable applications. Smart Materials and Structures 17.2 (2008): 025017.

[196] Tiersten, H.F. (1981). Electroelastic interactions and the piezoelectric equations. J. Acoust. Soc. Am. $70(6)$.

[197] R.A. Toupin, Theories of elasticity with couple-stress, Archive for Rational Mechanics and Analysis 17 (1964) 85-112.

[198] Turner, 1998. Three rules for bone adaptation to mechanical stimuli, Bone, 23(5): 399-407.

[199] Tzou, Horn S. Piezoelectric shells: distributed sensing and control of continua. London: Kluwer Academic, 1993.

[200] Tzou, H. S., and C. I. Tseng. Distributed piezoelectric sensor/actuator design for dynamic measurement/control of distributed parameter systems: a piezoelectric finite element approach. Journal of sound and vibration 138.1 (1990): 17-34.

[201] Tzou, H. S., and M. Gadre. Theoretical analysis of a multi-layered thin shell coupled with piezoelectric shell actuators for distributed vibration controls. Journal of Sound and Vibration 132.3 (1989): 433-450.

[202] Tzou, H. S., and J. P. Zhong. Electromechanics and vibrations of piezoelectric shell distributed systems. Journal of dynamic systems, measurement, and control 115.3 (1993): 506-517.

[203] Tzou, H. S., and H. Q. Fu. A study of segmentation of distributed piezoelectric sensors and actuators, part I: theoretical analysis. Journal of Sound and Vibration 172.2 (1994): 247-259.

[204] Yang, S. Y., and W. H. Huang. Is a collocated piezoelectric sensor/actuator pair feasible for an intelligent beam?. Journal of sound and vibration 216.3 (1998): 529-538.

[205] http://www.piceramic.de/pdf/PIC_Brochure_DuraAct_Piezo_Composite_Patch_Transducers_C2_pic.pdf

[206] F. Vestroni, F. dell'Isola, S. Vidoli, M.N. Cerri. Structural health monitoring based on dynamic measurements: A standard and a novel approach. System-Based Vision for Strategic and Creative Design, $1-3$ 2023-2028, 2003

[207] S. Vidoli and F. dell'Isola. Modal coupling in one-dimensional electromechanical structured continua. Acta Mechanica, 141(1-2), 2000, 37-50.

[208] S. Vidoli and F. dell'Isola. Vibration control in plates by uniformly distributed PZT actuators interconnected via electric networks. European Journal of Mechanics, A/Solids, 20, 2001, 435-456. 
[209] S. Vidoli and F. dell'Isola. Continously distributed control of plates by electric networks with PZT actuators. Conference in honour of K. Hutter in occasion of his 60th birthday, 2001, 92-110.

[210] S. Vidoli, F. dell'Isola, Modal coupling in one-dimensional electromechanical structured continua, Acta Mechanica 141 (2000) 37-50.

[211] Y. Yang, W.Y. Ching, A. Misra, Higher-order continuum theory applied to fracture simulation of nano-scale intergranular glassy film, Journal of Nanomechanics and Micromechanics 1 (2011) 60-71.

[212] Y. Yang, A. Misra, Micromechanics based second gradient continuum theory for shear band modeling in cohesive granular materials following damage elasticity, International Journal of Solids and Structures 49 (2012) 2500-2514.

[213] V.A. Yeremeyev, A.B. Freidin, L.L. Sharipova, The stability of the equilibrium of two-phase elastic solids, Journal of Applied Mathematics and mechanics (PMM) 71 (2007) 61-84.

[214] Wen, Yumei, Ping Li, and Shanglian Huang. Readout of a piezoelectric distributed sensing network embedded in concrete. 5th Annual International Symposium on Smart Structures and Materials. International Society for Optics and Photonics, 1998.

[215] Y. Yang, A. Misra, Higher-order stress-strain theory for damage modeling implemented in an elementfree Galerkin formulation, Computer Modeling in Engineering and Sciences 64 (2010) 1-36.

[216] S. Zouhdi, S. Ari, Alexey P. Vinogradov, Metamaterials and Plasmonics: Fundamentals, Modelling, Applications. New York Springer-Verlag (2008).

[217] Wolff, J. The Law of Bone Remodelling (1986) Translated by P. Maquet and R. Furlong. (translation of the German 1892 edition) Berlin, Heidelberg: Springer-Verlag.

[218] S. Y. Wu. Method for multiple-mode shunt damping of structural vibration using a single PZT transducer. Smart Structures and Materials 1998: Passive Damping and Isolation, 3327:159-168, 1998. 